Draft version September 14, 2016

Preprint typeset using $\mathrm{LAT}_{\mathrm{E}} \mathrm{X}$ style emulateapj v. 08/22/09

\title{
MEASUREMENT OF REDSHIFT SPACE POWER SPECTRUM FOR BOSS GALAXIES AND THE GROWTH RATE AT REDSHIFT 0.57
}

\author{
Zhigang Li $^{1,2, *}$, Y.P. Jing ${ }^{1,2, *}$, Penguie Zhang ${ }^{1,2, *}$, Dalong Cheng ${ }^{1}$ \\ Draft version September 14, 2016
}

\begin{abstract}
We present a measurement of two-dimensional (2D) redshift-space power spectrum for the Baryon Oscillation Spectroscopic Survey (BOSS) Data Release 11 CMASS galaxies in the North Galactic Cap (NGC) based on the method developed by Jing \& Börner (2001). In this method, we first measure the 2D redshift-space correlation function for the CMASS galaxies, and obtain the 2D power spectrum based on Fourier Transform of the correlation function. The method is tested with an N-body mock galaxy catalog, which demonstrates that the method can yield an accurate and unbiased measurement of the redshift-space power spectrum given the input $2 \mathrm{D}$ correlation function is correct. Compared with previous measurements in literature that are usually based on direct Fourier Transform in redshift space, our method has the advantages that the window function and shot-noise are fully corrected, while those measured in previous studies for the CMASS galaxies are usually the one convolved with the window function. In fact, our 2D power spectrum, by its construction, can accurately reproduce the $2 \mathrm{D}$ correlation function, and in the meanwhile can reproduce, for example, the $2 \mathrm{D}$ power spectrum of Beutler et al. (2014) accurately if ours is convolved with the window function they provided. Thus, our measurement can facilitate a direct comparison with the theoretical predictions. With this accurate measurement of the 2D power spectrum, we then develop a method to measure the structure growth rate, by separating the anisotropic redshift-space power spectrum from the isotropic real-space power spectrum. We have also carefully corrected for the nonlinearities in the mapping from real space to redshift space, according to the theoretical model of Zhang et al. (2013). Finally, we obtain $f\left(z_{\text {eff }}\right) \sigma_{8}\left(z_{\text {eff }}\right)=0.438 \pm 0.037$ at the effective redshift $z_{\text {eff }}=0.57$, where $f\left(z_{\text {eff }}\right)$ is the linear growth rate and $\sigma_{8}\left(z_{\text {eff }}\right)$ is the rms density fluctuation in the sphere of comoving radius $8 h^{-1} \mathrm{Mpc}$ at $z_{\text {eff }}$. The result is useful for constraining cosmological parameters. The measurements of $2 \mathrm{D}$ power spectrum will be released soon.
\end{abstract}

Subject headings: cosmology:observation, large-scale structure, galaxy survey

\section{INTRODUCTION}

Redshift space distortion (RSD) is emerging as a major probe of cosmology, and is playing an important role in ongoing and upcoming dark energy surveys (e.g. Feng et al. (2014); Dodelson et al. (2016)). Peculiar velocities of galaxies distort their distribution in redshift space through the Doppler effect. They render the otherwise statistically isotropic distribution of galaxies in real space into a statistically anisotropic distribution in redshift space with a unique pattern. Through such unique anisotropic pattern, in principle one is able to infer statistical properties of peculiar velocities at cosmological distances. These statistics depend on both the law of gravity, and the nature of gravitational sources (dark matter, dark energy, etc.). It then provides us a precious tool to measure the structure growth of the universe and to probe properties of dark energy and gravity (e.g. Amendola et al. (2005); Yamamoto et al. (2005); Jain \& Zhang (2008); Linder (2008); Wang (2008); Percival \& White (2009); White et al. (2009); Song \& Percival (2009); Jennings et al. (2011); Cai \& Bernstein (2012)). Furthermore, the combi-

\footnotetext{
${ }^{1}$ Center for Astronomy and Astrophysics, Department of Physics and Astronomy, Shanghai Jiao Tong University, 800 Dongchuan Road, Shanghai, 200240

2 IFSA Collaborative Innovation Center, Shanghai Jiao Tong University, Shanghai 200240, China

*zhigli@sjtu.edu.cn;ypjing@sjtu.edu.cn;zhangpj@sjtu.edu.cn
}

nation of weak lensing and RSD allows for a test of General Relativity (GR), insensitive to unknown galaxy bias and cosmic variances, through the $E_{G}$ method (Zhang et al. 2007; Reyes et al. 2010; Leonard et al. 2015; Pullen et al. 2015b,a; Blake et al. 2016).

A major challenge of RSD studies in the era of precision cosmology lies in its theoretical modelling, due to several nonlinear processes entangled in the redshiftspace clustering of galaxies. However, precision measurement of RSD also faces unresolved problems. RSD has been measured extensively using correlation function (Hamilton 1993; Fisher et al. 1994; Loveday et al. 1996; Ratcliffe et al. 1998; Peacock et al. 2001; Guzzo et al. 2008; Okumura et al. 2008; Cabré \& Gaztañaga 2009a,b; Beutler et al. 2012; Reid et al. 2012; Chuang et al. 2013; Reid et al. 2014; Ross et al. 2014; Samushia et al. 2014; Sánchez et al. 2014; Howlett et al. 2015; Alam et al. 2015; Okumura et al. 2016). It has also been measured through the redshift-space power spectrum (Park et al. 1994; Cole et al. 1995; Landy et al. 1996; Jing \& Börner 2001, 2004; Yamamoto et al. 2006, 2008, 2010; Blake et al. 2011; Hikage \& Yamamoto 2013; Oka et al. 2014; Beutler et al. 2014; Kanemaru et al. 2015; Gil-Marín et al. 2016a,b; Johnson et al. 2016) and bispectrum (Gil-Marín et al. 2015a,b, 2016b). The redshift-space power spectrum is more directly connected to the theory of large scale structure (LSS). However, its precision measurement faces two difficulties. 
One problem is that RSD effect is along different line-of-sights (LOS) for different galaxies, while Fourier transform tends to mix different LOSs. In early works, the power spectrum analysis was usually based on the parallel-plane approximation, that is, all galaxies in the survey share one unique LOS direction. Then one can rely on the Fast Fourier Transform (FFT) technique to accelerate the power spectrum calculation. However, the sky area covered by galaxy surveys becomes larger and larger. The parallel-plane approximation becomes less and less accurate and systematics introduced by the variation of LOSs in the survey becomes nonnegligible. One solution beyond the parallel-plane approximation was proposed by Yamamoto et al. (2006) (Y06 hereafter). It has been implemented in various recent galaxy surveys (Yamamoto et al. 2006, 2008, 2010; Hikage \& Yamamoto 2013; Oka et al. 2014; Beutler et al. 2014; Kanemaru et al. 2015; Gil-Marín et al. 2016a,b; Johnson et al. 2016). In the Y06 method, each galaxy pair shares a common LOS, which is further approximated as that of one galaxy in the pair. With this 'moving-LOS approximation', the pair summation can be implemented by two Fourier transforms and then can be accelerated by FFT. Nevertheless, this may introduce notable systematics on the hexadecapole power spectrum for wide galaxy surveys (Samushia et al. 2012; Yoo \& Seljak 2015). Another proposed solution is to decompose the 3D density field with the spherical harmonics and spherical Bessel functions, and is referred as $\mathrm{SFB}^{4}$ hereafter (Heavens \& Taylor 1995; Fisher et al. 1995; Tadros et al. 1999; Hamilton et al. 2000; Taylor et al. 2001; Tegmark et al. 2002, 2004; Percival et al. 2004; Erdoğdu et al. 2006; Lanusse et al. 2012; Leistedt et al. 2012; Rassat \& Refregier 2012; Lanusse et al. 2015). This decomposition keeps the LOS information (and therefore RSD information) exactly. However, the measured SFB power spectrum differs from the redshiftspace power spectrum predicted by most commonly used RSD models. Furthermore, it mixes clustering at different redshifts. Both bring inconveniences when one compares the data with models.

Another important issue in the power spectrum measurements is the deconvolution of window function. Unlike that in the correlation function, it is nontrivial to correct the window function in Fourier space. On one hand, the window function couples different Fourier modes. On the other hand, the window function introduces nonuniform distribution of $\mu=\hat{k} \cdot \hat{n}_{\mathrm{LOS}}$. Here, $\hat{k}$ is the unit wavenumber vector and $\hat{n}_{\mathrm{LOS}}$ is the unit LOS vector. Such non-uniform $\mu$ distribution may bias the measurement of power spectrum multipoles (Samushia et al. 2012; Yoo \& Seljak 2015).

In this paper, we propose to use the two-dimensional (2D) galaxy power spectrum in redshift space to measure the RSD effect, instead of using the multipole power spectrum or SFB coefficients. We revisit the method of measuring the 2D galaxy power spectrum through Fourier transform of the 2D galaxy correlation function in redshift space developed by Jing \& Börner (2001). This method improves the parallel-pane approximation

\footnotetext{
${ }^{4}$ In some literatures, it is called the spherical Fourier-Bessel expansion (SFB for short).
}

and the 'moving-LOS' approximation. In measurement of 2D correlation function, LOS is defined on each galaxy pair, usually to be the position vector of pair center with respect to the observer. The LOS defined in this way captures all information of RSD under the assumption of distant observer and neglecting wide-angle effect. Furthermore, the window function can be corrected in configuration space robustly and efficiently since the deconvolution in Fourier space becomes division in configuration space. The non-uniform $\mu$-distribution can also be solved by uniformly weighting the correlation function in $(s, \mu)$ space.

Next, we propose a method to measure the structure growth rate through the $2 \mathrm{D}$ power spectrum measurement. We separate the anisotropies on the galaxy power spectrum in redshift space from the isotropic galaxy power spectrum in real space by introducing a new statistics - anisotropic measure. When modeling the anisotropic measure, we have corrected for the nonlinearities with the theoretical model of Zhang et al (2013). In this way, the RSD parameter and galaxy bias can be measured independently. We apply the method to the BOSS-DR11 CMASS galaxy sample and obtain a robust measurement of the structure growth rate.

The paper is organized as follows. In $\S 2$, we introduce the method of measuring the $2 \mathrm{D}$ power spectrum in redshift space for a large galaxy survey and test it with a mock galaxy catalog based on an $N$-body simulation. In $\S 3$, we introduce the data set used in this paper: BOSSDR11 CMASS galaxy sample and the MD-Patchy mock galaxy catalogs. In $\S 4$, we show the measured $2 \mathrm{D}$ power spectrum of BOSS-DR11 CMASS galaxies. We measure the structure growth rate from the measured $2 \mathrm{D}$ power spectrum and compare it with previous studies in $\S 5$. We end the paper with a brief summary in $\S 6$.

\section{METHOD OF MEASURING REDSHIFT SPACE DISTORTION POWER SPECTRUM}

In this section, we describe our method to measure the 2D power spectrum from a large redshift survey. We will also verify this method against the mock galaxy distribution in a high-resolution simulation.

Peculiar velocity $\mathbf{v}$ of a galaxy adds a Doppler redshift on top of its cosmological redshift. Therefore, the realspace position at $\mathbf{r}$ changes to the corresponding position at $\mathbf{s}_{1}$ in redshift-space

$$
\mathbf{s}_{1}=\mathbf{r}+\left[\frac{\mathbf{v} \cdot \hat{r}}{H(z)}\right] \hat{r} .
$$

Each galaxy has its own LOS $(\hat{r})$ and therefore only the velocity component $(\mathbf{v} \cdot \hat{r})$ along the LOS contributes to RSD. Large surveys can have very different LOSs, so the variation of LOSs must be taken into account. A direct Fourier transform mixes all LOSs in the survey volume and therefore can erase most, if not all, RSD signal. In correlation function, this problem is much suppressed. For a pair of galaxies at redshift-space positions $\mathbf{s}_{1}$ and $\mathbf{s}_{2}$, we can decompose the separation vector $\mathbf{s} \equiv \mathbf{s}_{2}-\mathbf{s}_{1}$ into $\mathbf{s}=\left(\mathbf{s}_{\perp}, s_{\|}\right)$, where $s_{\|}$is the separation along the LOS pointing to the center of the pair $\mathbf{s}_{h} \equiv\left(\mathbf{s}_{1}+\mathbf{s}_{2}\right) / 2$ (that is $\left.s_{\|}=\mathbf{s} \cdot \hat{\mathbf{s}}_{h}\right)$ and $\mathbf{s}_{\perp}$ is the $2 \mathrm{D}$ transverse component of $\mathbf{s}$. We can then measure the correlation function $\xi^{s}\left(\mathbf{s}_{\perp}, s_{\|}\right)$. The superscript ' $s$ ' denotes redshift-space property. From symmetry argument, the 
correlation function depends on the amplitude of $\mathbf{s}_{\perp}$, but not its direction. Then the expectation value of the correlation function depends only on $s_{\perp}$ and $s_{\|}$. Therefore we often call $\xi^{s}\left(s_{\perp}, s_{\|}\right)$the $2 \mathrm{D}$ correlation function, or the anisotropic correlation function. Instead of approximating all LOSs as a single LOS in the direct Fourier transform, the correlation function measurement only requires that the two LOSs $\left(\mathbf{s}_{1,2}\right)$ of a given pair can be approximated as the LOS to the pair center $\left(\mathbf{s}_{h}\right)$. Therefore it is a much more accurate approximation. The accuracy is of the order $\theta^{2} / 2=1.5 \%\left(\theta / 10^{\circ}\right)^{2}$ where $\theta$ is the angular separation of the pair. For BOSS CMASS galaxies we analyze $\left(z_{\text {med }}=0.57\right)$ and for the scale $s_{\perp} \lesssim 300 h^{-1} \mathrm{Mpc}$ we are interested, the accuracy is better than $2 \%$. So we can neglect the error caused by this approximation ${ }^{5}$. Therefore the correlation function $\xi^{s}\left(s_{\perp}, s_{\|}\right)$faithfully captures the RSD effect.

Nevertheless, from the viewpoint of theoretical modeling and cosmological parameter fitting, the 2D power spectrum is more convenient. First, it is more directly connected to the theory of LSS. Second, it is more straightforward to cut in $(k, \mu)$ space to minimize uncertainties of cosmological parameter fitting, arising from various nonlinearities. Nonlinearities in real-space clustering can be mitigated by cut in $k$, while nonlinearities in real space-redshift space mapping can be mitigated by cut in $\mu$. The major goal of this paper is to test the method of measuring the 2D power spectrum and apply it on the BOSS-DR11 CMASS galaxies.

\subsection{Measurement of $2 D$ Power Spectrum}

Following Jing \& Börner (2001), we apply a correlation function based method to measure the anisotropic redshift-space power spectrum of BOSS galaxies. It is a two-step procedure. We first measure the twodimensional correlation function $\xi^{s}\left(s_{\perp}, s_{\|}\right)$. Then we translate the $2 \mathrm{D}$ correlation function to the $2 \mathrm{D}$ power spectrum by Fourier transform.

\subsubsection{Measuring the $2 D$ correlation function}

The redshift-space galaxy correlation function is measured using the Landy-Szalay estimator,

$$
\xi_{g}^{s}\left(s, \mu_{s}\right)=\frac{\mathrm{DD}-2 \mathrm{DR}+\mathrm{RR}}{\mathrm{RR}} .
$$

Notice that, instead of binning in $s_{\perp}-s_{\|}$space, we bin in $s-\mu_{s}$ space. Here, $s \equiv \sqrt{s_{\perp}^{2}+s_{\|}^{2}}$ and $\mu_{s}=s_{\|} / s$. DD represents the normalized number of galaxy-galaxy pairs whose separation lies in the corresponding $\left(s, \mu_{s}\right)$ bins, DR the number of galaxy-random pairs and RR the number of random-random pairs. The normalizations are taken as the total number of pairs for each component, i.e. $N_{g}\left(N_{g}-1\right) / 2$ for DD, $N_{g} N_{R}$ for DR and $N_{R}\left(N_{R}-1\right) / 2$ for $R R$ with total number of galaxies $N_{g}$ and total number of random points $N_{R}$. The number of random points are taken to be about 100 times of the number of galaxies which is large enough for the correction of window function.

\footnotetext{
${ }^{5}$ The wide-angle effect has been shown to be small in SDSS-like galaxy surveys and is expected be even smaller in the BOSS-like galaxy surveys (Samushia et al. 2012; Yoo \& Seljak 2015)
}

We use adaptive bin size in $s$-direction to reach high resolution on small scales and maintain reasonable signalto-noise ratio on large scales simultaneously. We have a total of 36 bins in the range of $0<s<300 h^{-1} \mathrm{Mpc}$ : two bins are in the range of $0-2 h^{-1} \mathrm{Mpc} ; 3$ bins in $2-8 h^{-1} \mathrm{Mpc} ; 4$ bins in $8-24 h^{-1} \mathrm{Mpc} ; 17$ bins in $24-160 h^{-1} \mathrm{Mpc} ; 10$ bins in $160-300 h^{-1} \mathrm{Mpc}$. The bin sizes within each range are equal, which are $1 h^{-1} \mathrm{Mpc}$, $2 h^{-1} \mathrm{Mpc}, 4 h^{-1} \mathrm{Mpc}, 8 h^{-1} \mathrm{Mpc}$ and $14 h^{-1} \mathrm{Mpc}$, respectively.

In the $\mu_{s}$-direction, we use 20 bins with equal size of $\Delta \mu_{s}=0.05$. We have checked that differences of binning in $\left(s, \mu_{s}\right)$ space result in negligible changes in the measured power spectrum. In the end, we interpolate the resulting $2 \mathrm{D}$ correlation function $\xi^{s}\left(s, \mu_{s}\right)$ on fine grids of $\left(s_{\perp}, s_{\|}\right)$space for calculation of the $2 \mathrm{D}$ power spectrum in the next step, where $s_{\|}=s \cdot \mu_{s}$ and $s_{\perp}=\sqrt{s^{2}-s_{\|}^{2}}$.

\subsubsection{Measuring the $2 D$ power spectrum}

From the 2D correlation function, we calculate the 2D power spectrum using the Fourier transform,

$$
\begin{aligned}
P_{g}^{s}(k, \mu) & =\int \xi_{g}^{s}\left(s_{\perp}, s_{\|}\right) e^{i \mathbf{k} \cdot \mathbf{s}} d^{3} \mathbf{s} \\
& =\int \xi_{g}^{s}\left(s_{\perp}, s_{\|}\right) e^{i\left(k_{\|} s_{\|}+k_{\perp} s_{\perp} \cos (\phi)\right)} s_{\perp} d s_{\perp} d \phi d s_{\|} \\
& =\int \xi_{g}^{s}\left(s_{\perp}, s_{\|}\right) K\left(k_{\perp}, k_{\|} ; s_{\perp}, s_{\|}\right) s_{\perp} d s_{\perp} d s_{\|},
\end{aligned}
$$

where $k_{\|}=k \cdot \mu$ and $k_{\perp}=\sqrt{k^{2}-k_{\|}^{2}}$. Notice that $\mu$ here is not related to $\mu_{s}$ in the correlation function. The kernel $K$ is defined as $K\left(k_{\perp}, k_{\|} ; s_{\perp}, s_{\|}\right)=$ $\cos \left(k_{\|} s_{\|}\right) J_{0}\left(k_{\perp} s_{\perp}\right)$ with $J_{0}(x)=\int e^{i x \cos (\phi)} d \phi$ the zeroth order Bessel function. In practice, we need to cut the integral at some maximum value $s_{\max }$ to avoid contaminations from poor data at large $s$. But too small a value of $s_{\max }$ will introduce significant bias on the power spectrum, i.e. suppress power on large scales. For BOSSDR11 CMASS galaxy sample that we will analyze later, we have tested that $s_{\max }=300 h^{-1} \mathrm{Mpc}$ is an appropriate choice without introducing notable bias. The computational cost to calculate $2 \mathrm{D}$ correlation function up to this $s_{\max }$ is about 48 hours on a workstation for BOSSDR11 CMASS NGC galaxies with 100 times more random points. For mock samples, we use 10 times more random points than mock galaxies. So we only need 24 CPU hours to measure 2D correlation function for one mock sample. by

The multipoles of $2 \mathrm{D}$ power spectrum can be calculated

$$
P_{g, l}^{s}(k)=(2 l+1) \int_{0}^{1} P_{g}^{s}(k, \mu) \mathcal{L}_{l}(\mu) d \mu .
$$

The first few Legendre polynomials we used are, $\mathcal{L}_{0}(x)=$ $1, \mathcal{L}_{2}(x)=\left(3 x^{2}-1\right) / 2$ and $\mathcal{L}_{4}(x)=\left(35 x^{4}-30 x^{2}+3\right) / 8$.

\subsection{Test with an $N$-body simulation}

We test our redshift-space power spectrum measurements using mock galaxies constructed from an $N$ body simulation, where we can build redshift-space mock 


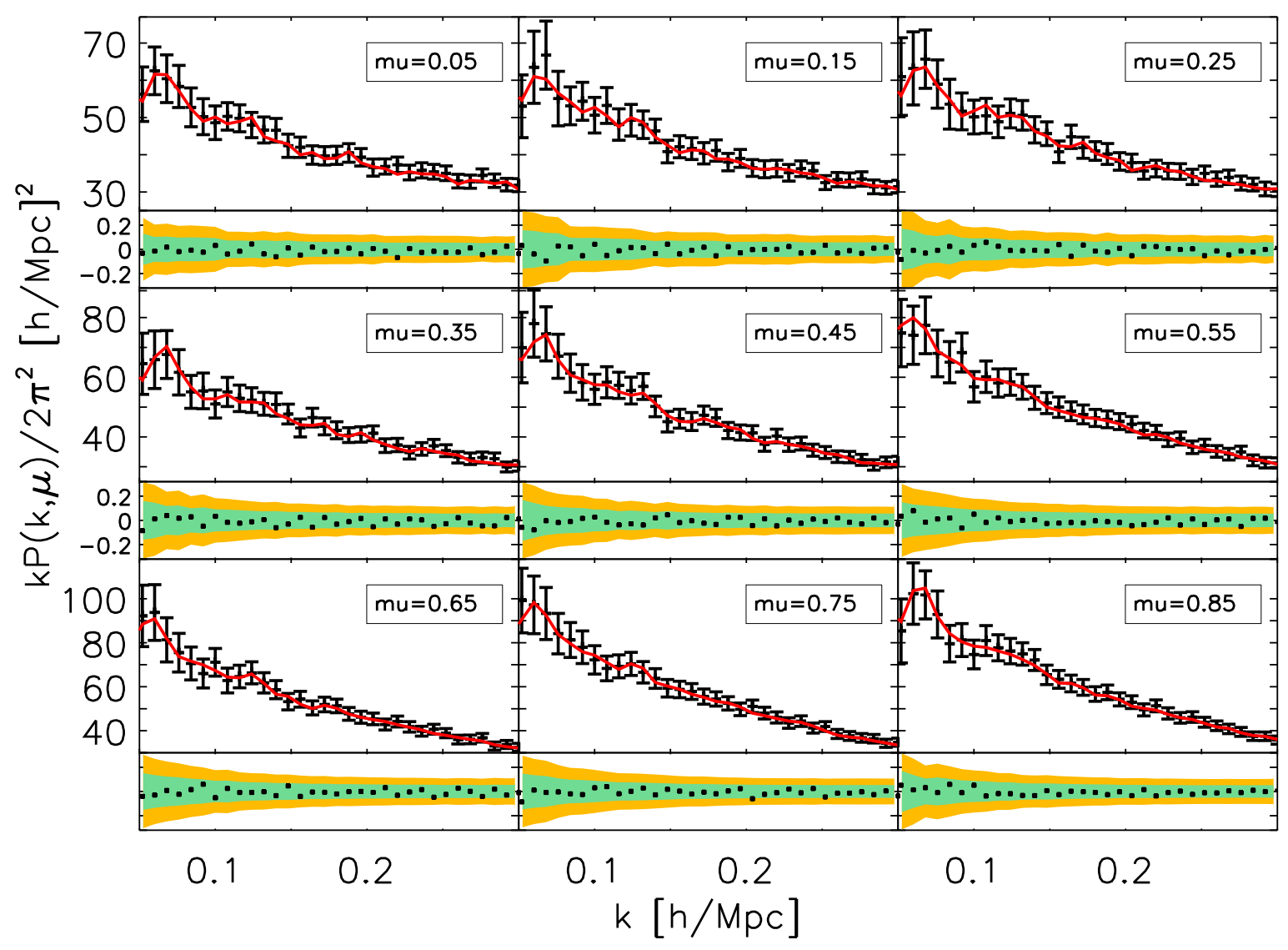

FIG. 1. - 2D power spectrum of mock galaxies in the CosmicGrowth simulation. The nine panels show $k P(k, \mu) / 2 \pi^{2}$ distribution as a function of $k$, for specific $\mu$ bins. The power spectrum we obtained with our method are shown as red solid lines and those using FFT method are shown as black plus signs in the top sub-box of each panel. The error bars for the data points of FFT method are estimated according to Eq. 6 . In the bottom sub-box of each panel shows the difference between the two methods, $\left(P(k, \mu)-P_{\mathrm{FFT}}(k, \mu)\right) / P_{\mathrm{FFT}}(k, \mu)$. The Celadon green (Chrome yellow) band shows $1 \sigma(2 \sigma)$ confidence level. The two methods show good consistency. The differences between them are well within the $1 \sigma$ level.

galaxy distribution without wide-angle effect. This allows us to make an exact comparison between our method and the FFT method.

The simulation we used is one of high-resolution CosmicGrowth simulations (Jing, in preparation), which were generated by the $\mathrm{P}^{3} \mathrm{M}$ code of Jing et al. (2007) with $3072^{3}$ particles contained in a box of $1.2 h^{-1} \mathrm{Gpc}$ on a side. The initial condition is made at redshift $z_{i}=144$ following the Zeldovich approximation with the transfer function from Seljak \& Zaldarriaga (1996). The cosmological parameters are set as $\Omega_{m}=0.268, \Omega_{b}=0.044$, $\Omega_{\Lambda}=0.732, n_{s}=0.96$ and $\sigma_{8}=0.83$. The simulation run 5000 even steps in the expansion factor to redshift $z=0$. We further made the host halo catalog through the Friend-Of-Friend (FOF) method with a link parameter $b_{\text {link }}=0.2$. The sub-halos are identified using the Hierarchical-Bound-Tracing (HBT) algorithm (Han et al. 2012).

We relate galaxies to sub-halos and infer the stellar mass for galaxies using the subhalo-galaxy matching method proposed by Wang et al. (2006),

$$
M_{\text {star }}=\frac{2 c}{\left(M_{\text {infall }} / M_{0}\right)^{-a}+\left(M_{\text {infall }} / M_{0}\right)^{-b}}
$$

where the infall mass $M_{\text {infall }}$ is defined as the mass of the sub-halo at the time when it was last the central dom- inant object. Then we introduce a Gaussian scatter on $\log \left(M_{\text {star }}\right)$ at a given value of $M_{\text {infall }}$ with dispersion $\sigma_{M}$. We adopt the parameters given in Wang \& Jing (2010) which can fit the stellar mass function and correlation function of SDSS galaxies at $z=0$ and VVDS galaxies at $z=0.83$ simultaneously: $a=0.29, b=2.42, c=$ $10^{10.15} h^{-1} M_{\odot}, M_{0}=4.34 \times 10^{11} h^{-1} M_{\odot}$ and $\sigma_{M}=0.24$. We use a stellar mass threshold of $10^{10.98} M_{\odot}$ to obtain number density of $\bar{n}_{\text {gal }}=4 \times 10^{-4}(\mathrm{Mpc} / h)^{-3}$ (which is the observed peak density at $z=0.5$ ) for mock galaxies. The mock galaxy catalog has a bias of $b_{\text {gal }}=1.68$.

In order to convert the real-space position (box coordinates) to redshift-space coordinate, we assume a distant observer in the $\hat{\mathbf{z}}$ direction, obtaining $s=r+$ $v_{z} / a H$. Where $v_{z}$ is the physical peculiar velocity along $z$-direction and the value of $a H$ is taken at redshift $z=0.57$. To enhance the $\mathrm{S} / \mathrm{N}$ ratio, this procedure is also applied to the $\hat{\mathbf{x}}$ and $\hat{\mathbf{y}}$ directions. In the end, power spectrum is averaged over those obtained for each specific direction (observer).

Next, we measure the 2D power spectrum in redshift space for the resulting mock galaxy catalog, following the same procedure described in Sec. 2.1. We also use the FFT method to measure the redshift-space 2D power spectrum for the same mock galaxy catalog. The size of the FFT box is $1200 h^{-1} \mathrm{Mpc}$ at one side. We use $1200^{3}$ 
grids on which the galaxy densities are calculated and Fourier transformed to get the power spectrum. For the FFT method, we estimate the errors of the 2D power spectrum by assuming a Gaussian distribution,

$$
\sigma_{P}=\sqrt{\frac{2}{N_{\text {mode }}(k, \mu)}}\left(P^{s}(k, \mu)+\frac{1}{\bar{n}_{\text {gal }}}\right)
$$

where $N_{\text {mode }}(k, \mu)$ is the number of modes residing in the $(k, \mu)$-bin, $\bar{n}_{\text {gal }}$ is the mean number density of mock galaxies and $P^{s}(k, \mu)$ is the power spectrum of mock galaxies. The first term in the right-hand side is the cosmic variance and the second term is shot-noise.

The measured 2D power spectrums of mock galaxies are shown in Fig. 1. The 9 panels show $k P^{s}(k, \mu) / 2 \pi^{2}$ for $9 \mu$-bins. We also show the difference between the two methods, $\left(P^{s}(k, \mu)-P_{\mathrm{FFT}}^{s}(k, \mu)\right) / P_{\mathrm{FFT}}^{s}(k, \mu)$, in the bottom sub-box in each panel. Where $P^{s}(k, \mu)$ stands for the $2 \mathrm{D}$ power spectrum measured using our method and $P_{\mathrm{FFT}}^{s}(k, \mu)$ for the direct FFT method. The two methods show good consistency over almost all scales. The differences between them are well within $1 \sigma$ level. Although they are omitted, the $2 \mathrm{D}$ power spectrums at $\mu=0.95$ bin are consistent at the same level as others. Therefore we verify that obtaining the $2 \mathrm{D}$ power spectrum from the $2 \mathrm{D}$ correlation function is unbiased. Together with the sophisticated and accurate method of measuring the $2 \mathrm{D}$ correlation function, we conclude that the 2D power spectrum measured in this way can faithfully capture the RSD effect.

Furthermore, the 2D power spectrum based on the Fourier transform of the2D correlation function has a few advantages:

- The 2D power spectrum is free of normalization and shot-noise subtraction, since the correlation function is free of such issues.

- The survey window function is dealt with in configuration space when measuring the $2 \mathrm{D}$ correlation function, thus avoiding the deconvolution problem in the traditional multipole power spectrum measurements. It has been shown that the decoupling of window function in configuration space is stable and efficient.

- The nonuniform distribution of cosine angle $\mu$ can be solved when measuring 2D power spectrum multipoles (Eq.4). The main difference between our method and the traditional multipole power spectrum measurements is the way of weighting the data. In the traditional multipole power spectrum measurements, each galaxy or galaxy pair has equal weight. However, the $\mu$-distribution of galaxy pairs is commonly nonuniform in realistic galaxy surveys and so introduces systematics on the measured multipole power spectrum. We apply equal weight to the $2 \mathrm{D}$ correlation function where the $\mu$-distribution can be sufficiently uniform. It is worth noting that the method of Y06 without the 'moving-LOS approximation' is formally equivalent to the method used in this paper to measure the multipole power spectrum.
- The wide-angle effect can be reduced. The 2D correlation function at large $s$-scales usually has low signal-to-noise ratio and contains little cosmological information. When calculating the 2D power spectrum from the 2D correlation function, we would cut the integral at some maximum value of $s_{\max }$ to prevent the contamination of poor data. This effectively reduces the impact of wide-angle effect which is important only at large separations.

\section{DATA SET}

\subsection{BOSS-DR11 CMASS Galaxies}

In this paper, we use the publicly released CMASS galaxy sample in the Data Release 11 of Sloan Digital Sky Survey III (SDSS III) Baryon Oscillations Spectroscopic Survey (BOSS). SDSS has scanned over one third of the sky and obtained images in five photometric bandpasses to a limiting magnitude of $r_{\text {lim }} \approx 22.5$ using the 2.5 meter Sloan Telescope located at Apache Point Observatory in New Mexico. As a part of SDSS III, BOSS is designed to do spectroscopic observations of more than one million galaxies covering 10000 square degrees on the sky. Within BOSS, the CMASS sample is approximately stellar-mass limited above $z=0.45$, and target galaxies are selected from SDSS DR8 imaging data with selection function described in Anderson et al. (2014). The median redshift of CMASS galaxies is at $z_{\text {med }} \approx 0.57$ and the stellar mass peaks at $M_{\text {stellar }} \approx 10^{11.3} M_{\odot}$. Most of the CMASS galaxies are central galaxies in dark matter halos of mass about $10^{13} h^{-1} M_{\odot}$ with a non-negligible fraction of satellites which reside in halos about 10 times more massive. The BOSS-DR11 CMASS sample contains 690,826 galaxies and covers 8498 square degrees. We limit our analysis to the North Galactic Cap (NGC) sample which contains 520,805 galaxies and covers 6769 square degrees.

We correct for the effects of redshift failure and fiber collision by up-weighting the galaxies whose nearest neighbor had a redshift failure $\left(w_{\mathrm{rf}}\right)$ or failed to get redshift because they are a close pair $\left(w_{\mathrm{cp}}\right)$. We also apply the systematic weights to account for the seeing effect $\left(w_{\text {seeing }}\right)$ and correlation between the number density of observed galaxies and the stellar density $\left(w_{\text {star }}\right)$. All of these weights are documented in the publicly released data. To reach minimum variance for galaxy clustering measurement, we apply the FKP weight (Feldman et al. 1994) in a simple form, $w_{\mathrm{FKP}}(\mathbf{r})=1 /\left(1+\bar{n}(\mathbf{r}) P_{0}\right)$ with $P_{0}=20000$, where $\bar{n}(\mathbf{r})$ is the expected galaxy number density. The total weight applied to each galaxy is then,

$$
w_{\text {tot }}=\left(w_{\mathrm{rf}}+w_{\mathrm{cp}}-1\right) w_{\text {seeing }} w_{\text {star }} w_{\mathrm{FKP}}
$$

The survey completeness has been carefully calculated and publicly released together with the catalog. The random points are generated following the survey completeness in light of the 'Mangle' software (Hamilton \& Tegmark 2004; Swanson et al. 2008). The redshifts and FKP weights are 'shuffled' (e.g., randomly selected) from the observational sample to be assigned to the random points following a uniform distribution (Ross et al. 2012). Please refer to Anderson et al. (2014) for more details about the BOSS-DR11 CMASS galaxy sample and Ross et al. (2012) for the effect of the various weights. 


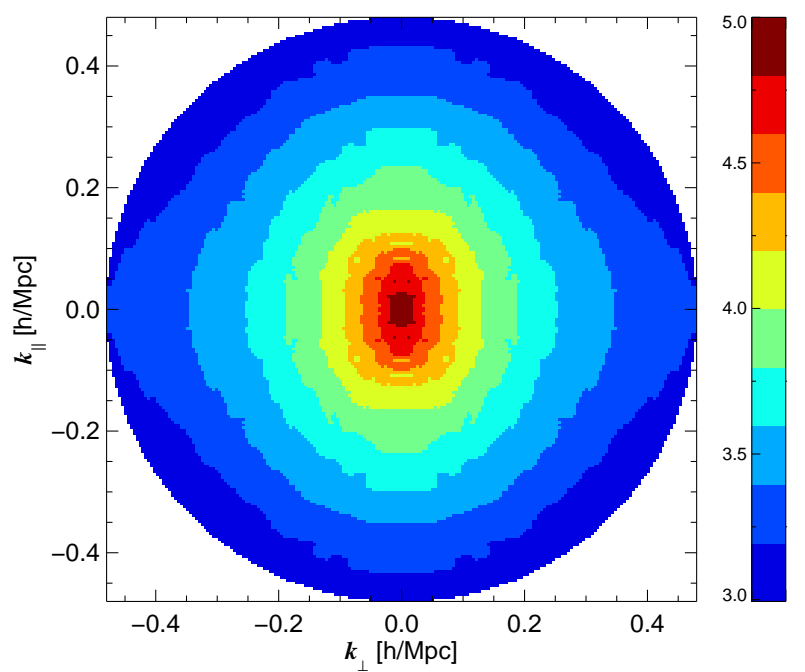

FIG. 2.- Illustration of the 2D power spectrum of galaxies in BOSS-DR11 CMASS sample. We present a color map of the $\log _{10} P\left(k_{\perp}, k_{\|}\right)$as a function of $k_{\perp}$ and $k_{\|}$. In the absence of RSD effect, we would obtain perfect circles with this discrete color scale. In the $k_{\|}$direction, we clearly see in the center the elongation due to the Kaiser effect, and in outskirts the squashing due to the FOG effect.

For this observational sample, we assume a $\Lambda$ CDM cosmology to transfer the redshift to comoving distance with parameters, $\Omega_{m}^{\text {fid }}=0.274, \Omega_{\Lambda}^{\text {fid }}=0.726$.

\subsection{Mock Catalogs to measure the covariance matrix}

To calculate covariance matrix for the $2 \mathrm{D}$ power spectrum and anisotropic measure defined in the following section, we use the MultiDark Patchy mock catalogs (hereafter MD-Patchy mocks) for BOSS DR11 CMASS sample (Kitaura et al. 2016). The MD-Patchy mocks are constructed relying on the PATCHY approximate simulations of dark matter density fields and using a biasing model to populate galaxies in the dark matter density fields (Kitaura \& Heß 2013; Kitaura et al. 2014, 2015). A coherent peculiar velocity field is calculated using the augmented Lagrangian Perturbation Theory (ALPT) consistently with the displacement field. The finger-of-god (FOG) effect is modeled using a Gaussian distribution function with parameters calibrated on the monopole and quadrupole damping effect in BigMultiDark simulation and BOSS observational data. The MDPatchy mock catalogs are constructed assuming a $\Lambda$ CDM Planck cosmology: $\Omega_{m}^{\mathrm{MD}}=0.307115, \Omega_{b}^{\mathrm{MD}}=0.048206$, $\sigma_{8}^{\mathrm{MD}}=0.8288, n_{s}^{\mathrm{MD}}=0.9611$ and $h^{\mathrm{MD}}=0.6777$. The resulting MD-Patchy mocks reproduce the number density, selection function, survey geometry, multipole power spectrum, multipole correlation function and three point statistics of the BOSS DR11 CMASS sample. The MDPatchy mock catalogs have been tested and applied on the analysis of BOSS galaxy surveys (Chuang et al. 2013, 2016; Cuesta et al. 2016).

\section{RESULTS OF POWER SPECTRUM MEASUREMENT}

In Fig. 2, we show the 2D power spectrum $P_{g}^{s}\left(k_{\perp}, k_{\|}\right)$ for BOSS-DR11 CMASS galaxies, measured using our method. In order to highlight the features, we collect the value of the $2 \mathrm{D}$ power spectrum into 10 equal bins. The 'elongated' feature in the central region is due to the

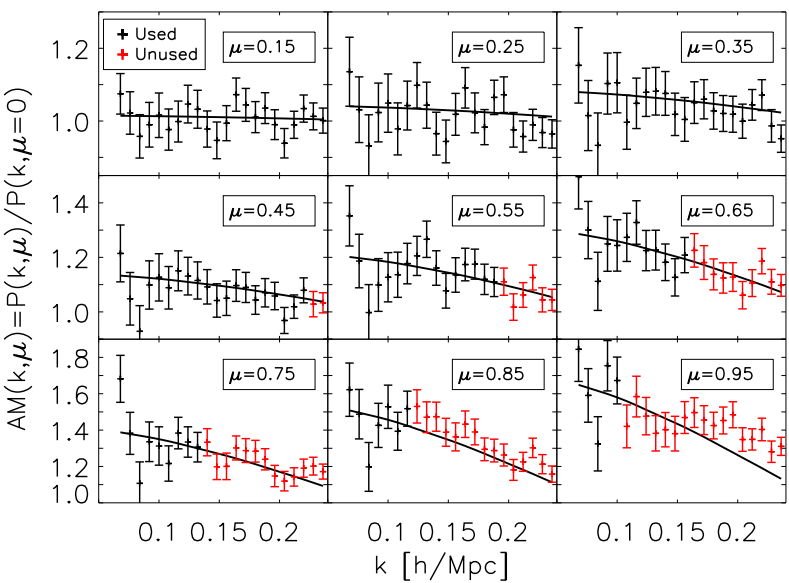

FIG. 3.- Power spectrum anisotropies of galaxies in BOSSDR11 CMASS sample. Each panel shows the anisotropic measure $\operatorname{AM}(k, \mu)$ for one $\mu$ bin. Our measurements are indicated with the plus symbols and our best fit model (see Eq. 12) with the solid lines. The errors are estimated using 1024 MD-Patchy mocks. Data points, with $k \mu<0.1 \mathrm{hMpc}^{-1}$, used in the fitting are indicated in black, while unused data points are indicated in red.

large-scale (small $k$-value) Kaiser effect (Kaiser 1987). The 'squashing' feature can also be seen in the outskirts, where the FOG effect defeats the Kaiser effect.

Although our method has been verified against mock galaxies in the $N$-body simulation $(\S 2.2)$, we further perform two consistency tests against published BOSS measurements, to make sure that we have understood the BOSS data correctly and have applied our method correctly. These consistency tests are presented in the appendix. There we have shown that our measured 2D power spectrum can reproduce very well the correlation functions of Anderson et al. (2014) and Sánchez et al. (2014). This is not surprising by the construction, but it does indicate that our measurement for the $2 \mathrm{D}$ power spectrum is unbiased for the systematics such as the window function and shot noises. We have also shown that our 2D power spectrum, once convolved with the window function provided by Beutler et al. (2014), can very accurately reproduce their power spectrum monopoles and quadrupoles as well, which further lends the support to our method.

One might worry about the possible coupling between different modes on the 2D power spectrum measurement since we rely on Fourier transforming the correlation function where the mode-coupling effect might be strong. In practice, we find that the resulting correlations between different modes using our method are weak. This can be understood since the window function has been decoupled in the measurement of 2D correlation function which reduces the mode-coupling effect. We show the correlation coefficients measured from 1024 MD-Patchy mocks in Fig. 4, which is defined as,

$$
r_{a b}^{\mathrm{C}}=\frac{C_{i j, k l}}{\left\langle P_{g}^{s}\left(k_{i}, \mu_{j}\right)\right\rangle\left\langle P_{g}^{s}\left(k_{k}, \mu_{l}\right)\right\rangle}
$$

The covariance matrix of the $2 \mathrm{D}$ power spectrum $C_{i j, k l}=$ $\left\langle\left(P_{g}^{s}\left(k_{i}, \mu_{j}\right)-\bar{P}_{g}^{s}\left(k_{i}, \mu_{j}\right)\right)\left(P_{g}^{s}\left(k_{k}, \mu_{l}\right)-\bar{P}_{g}^{s}\left(k_{k}, \mu_{l}\right)\right)\right\rangle$ and $\bar{P}_{g}^{s}(k, \mu)=\left\langle P_{g}^{s}(k, \mu)\right\rangle$ is the mean power spectrum. The bracket ' \langle\rangle ' represents the ensemble average, which is estimated using 1024 MD-Patchy mocks in this work. The 


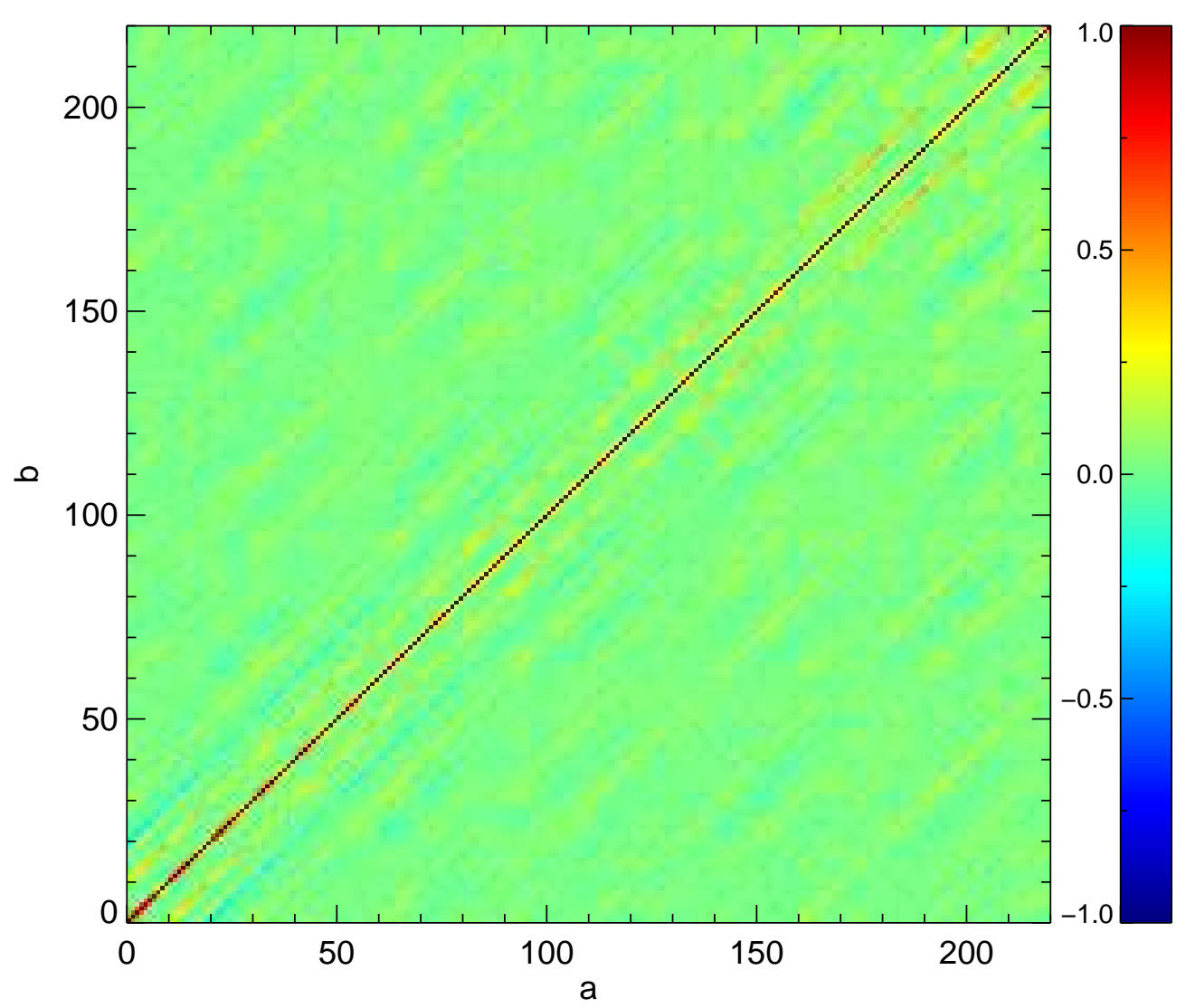

FIG. 4.- Correlation matrix of the 2D power spectrum for BOSS11-CMASS NGC galaxy sample, defined in Eq. 8. The colors indicate correlation level. Red color stands for high correlation, blue for high anti-correlation and green for no-correlation. In the figure shows 220 modes in total $(10 \mu \times 22 k)$, that is $a, b=0, \ldots, 219$.

index $a, b$ is related to $k$-bin index and $\mu$-bin index as, $a=i \times N_{\mu}+j$ and $b=k \times N_{\mu}+l$, where $i, k=0, \ldots, N_{k}-1$ and $j, l=0, \ldots, N_{\mu}-1$. We have $10 \mu$ bins $\left(N_{\mu}=10\right)$ and $22 k$ bins $\left(N_{k}=22\right)$ shown in Fig. 4 . The size of $k$ bin is $\Delta k=0.008 h^{-1} \mathrm{Mpc}$.

The goal of $2 \mathrm{D}$ power spectrum measurement is to infer the information of peculiar velocities. However, not all $P_{g}^{s}\left(k_{\perp}, k_{\|}\right)$measurement contain such useful information. Next we introduce a simple statistics, called the anisotropic measure (AM for short), to isolate the part useful for peculiar velocity and cosmology inference. This is motivated by a neat feature of $P_{g}^{s}\left(k_{\perp}, k_{\|}\right)$, that $P_{g}^{s}\left(k_{\perp}, k_{\|}=0\right)=P_{g}^{s}(k, \mu=0)$ is unaffected by RSD and equals the real-space power spectrum $P_{g}\left(k=k_{\perp}\right)$. Therefore all information of peculiar velocity is encoded in the ratio

$$
\operatorname{AM}(k, \mu) \equiv \frac{P_{g}^{s}(k, \mu)}{P_{g}^{s}(k, \mu=0)} .
$$

AM describes the anisotropies of galaxy clustering induced by RSD. In contrast, the denominator $P_{g}^{s}(k, \mu=$ 0 ) only contains the information of real-space clustering. Furthermore, its modeling is complicated by not only the nonlinear density evolution, but also the scale dependence and nonlinearities in galaxy bias. Therefore for cosmological constraints from RSD, it is better to work on $\operatorname{AM}(k, \mu)$ than on the full $P_{g}^{s}(k, \mu)$. In practice, we approximate the averaged $P_{g}^{s}(k, \mu)$ over $0.0<\mu<0.1$ as $P_{g}^{s}(k, \mu=0)=P_{g}(k)$, since for large $k$ of our interest, the RSD effect is negligible at $0.0<\mu<0.1$.

Since we Fourier transform correlation function to obtain $P_{g}^{s}(k, \mu)$ (and $\left.\operatorname{AM}(k, \mu)\right)$, numerically we can get a continuous series of data points in $(k, \mu)$ space. However, the number of independent modes is limited by the survey volume and the value of $s_{\max }$. The smallest dimension of CMASS sample is about $600 \mathrm{Mpc}(z$ from 0.43 to $0.70)$, corresponding to $k \sim 0.01 \mathrm{hMpc}^{-1}$. Moreover, the integral upper limit is adopted as $s_{\max }=300 h^{-1} \mathrm{Mpc}$, which sets a minimum value of $k$ to $\sim 0.02 \mathrm{hMpc}^{-1}$. The signal-to-noise ratio on large scales is determined by cosmic variance $(\mathrm{CV}), \mathrm{CV} \propto \sqrt{1 / N(k, \mu)}$. Here $N(k, \mu)$ is the number of modes in the $(k, \mu)$ bin. This means that for $k$ close to $0.02 h^{-1} \mathrm{Mpc}$, we do not have information to further split into $\mu$ bins to infer RSD. Therefore, in parameter fitting of $2 \mathrm{D}$ power spectrum in next section, we limit our analysis on scales of $k \geq 0.064 h / \mathrm{Mpc}$. We set the $k$-bin size to be $\Delta k=0.008 h / \mathrm{Mpc}$ and $\mu$-bin size to be $\Delta \mu=0.1$.

We show $\operatorname{AM}(k, \mu)$ for BOSS-DR11 CMASS galaxies in Fig. 3, but only data points with $k<0.24 h \mathrm{Mpc}^{-1}$ are shown. Each panel shows $\operatorname{AM}(k, \mu)$ for each $\mu$-bin. For $\mu$ bins of small value, the $\mathrm{RSD}$ effect $(\mathrm{AM} \neq 1)$ is insignificant. But for $\mu$ bins of large value, the RSD effect is significant. In particular we find that $\operatorname{AM}(k, \mu)>1$ for all modes shown in Fig. 3, meaning the Kaiser effect dominates over the FOG effect. But decreasing $\operatorname{AM}(k, \mu)$ with 
increasing $k$ does show the increasing impact of FOG.

\section{ESTIMATION OF THE STRUCTURE GROWTH RATE}

Since RSD is induced by peculiar velocity and peculiar velocity is related to matter density by the continuity equation, it allows us to measure a specific combination of the structure growth rate, $f D$ (or $\left.f(z) \sigma_{8}(z)\right)$. Here, $D$ is the linear density growth factor and $f \equiv d \ln D / d \ln a$. However, in reality it is highly nontrivial to constrain $f(z) \sigma_{8}(z)$ from RSD, due to various complexities in the RSD modeling (Zhang et al. 2013). We do not aim to take into account of all these complexities in our cosmological parameter fitting. Since the nonlinearities are only important at large $k$ and large $\mu$, it is possible to suppress their effects by selecting data in the $(k, \mu)$ space where the quasi-linear approximation is understood and works well. A data modeling including full nonlinearities will be investigated in a future work.

\subsection{RSD modeling}

We adopt the RSD model constructed by Zhang et al. (2013). The redshift-space galaxy power spectrum can be written as,

$$
\begin{aligned}
P_{g}^{s}(k, \mu)= & \left\{P_{g}(k)\left(1+\beta W(k) \mu^{2}\right)^{2}+\text { h.o. }\right\} \\
& \times \exp \left\{-\left(k \mu \tilde{\sigma}_{v}\right)^{2}\right\}
\end{aligned}
$$

Here $P_{g}(k)$ is galaxy power spectrum in real space. $\beta=f / b_{g}$ is the RSD parameter with $f$ the growth rate and $b_{g}$ the deterministic galaxy bias. The FOG effect in this formalism has been derived to have a Gaussian form, with $\tilde{\sigma}_{v}$ the velocity dispersion in unit of $H(z)$. The Gaussian form has been further verified in $N$-body simulations (Zheng et al. 2013).

This formula contains two kinds of corrections to the commonly adopted Kaiser plus FOG formula. The leading order correction is captured by $W(k)=$ $P_{g \theta}(k) / \beta P_{g}(k)$. It takes the nonlinear evolution of density-velocity relation into account and therefore the extends the Kaiser formula to nonlinear regime. $P_{g \theta}(k)$ is the density-velocity cross power spectrum and $\theta \equiv-\nabla$. $\mathbf{v} / H(z)$. In the limit of large scales density and velocity are perfectly correlated, so $W(k)=1$. Stochasticities develop in the density-velocity relation towards smaller scales and drives $W(k)<1$. Eventually $W(k) \rightarrow 0$ at deeply nonlinear region (Zheng et al. 2013).

All high order corrections are collected into one term 'h.o.', with the exact expressions given in Zhang et al. (2013). In principle, one would include all of the nonlinear terms to fit observed data. In this work, we take a different way to suppress the contribution of high-order terms by cutting data in $(k, \mu)$ space. This is motivated by the fact that we do not have a good understanding of nonlinearities and FOG effect which are very important to extracting the RSD parameter $\beta$ from galaxy clustering measurements. Although the FOG effect can be well described by a Gaussian damping function, the value of $\tilde{\sigma}_{v}$ is sensitive to how many nonlinear terms are included in the RSD models. Even though the full leading-order terms are included, the needed $\tilde{\sigma}_{v}$ for fitting the dark matter power spectrum still differs significantly from the velocity dispersion directly measured from the simulation (Zheng \& Song 2016). The authors of Zheng \& Song (2016) suggest that higher-order terms or including multi-streaming effect may explain the differences. This situation could be worse for galaxies since the galaxy bias will enhance the contribution of nonlinear terms.

Good news is that the nonlinear corrections are important only at large $k$ and large $\mu$. If we restrict our analysis only at small $k$ and small $\mu$, the effect of high-order terms can be reduced greatly. Then the FOG effect and RSD parameter can be obtained faithfully. In this first analysis, we choose a strict limit on the available data, that is, we only include data points with $k \mu \leq 0.1 \mathrm{hMpc}^{-1}$ and $k \leq 0.24 h \mathrm{Mpc}^{-1}$. This allows us to neglect the highorder correction terms 'h.o.' in Eq. 10 (Zheng \& Song 2016). We caution the readers that even with such strict cut, we still need to include $W(k)$, the effect of which is significant even at $k \sim 0.1 h / \mathrm{Mpc}$. We adopt a fitting formula for $W(k)$ given in Zheng et al. (2013),

$$
W(k)=1 /\left[1+\Delta \alpha(z) \Delta_{\mathrm{NL}}^{2}(k, z)\right]
$$

where $\Delta_{\mathrm{NL}}^{2}=k^{3} P_{m}(k) / 2 \pi^{2}$ is the nonlinear matter power spectrum. In practice, we use the mean value of $P_{g}^{s}(k, \mu=0)$ from 1024 mock samples divided by square of galaxy bias to estimate $P_{m}(k)$. The parameter $\Delta \alpha$ is also taken from Zheng et al. (2013) which is 0.376 at redshift $z=0.5$.

Furthermore, instead of fitting against the measured $P_{g}^{s}(k, \mu)$, we fit against the anisotropic measure defined in Eq. 9 using the following model,

$$
\operatorname{AM}^{\mathrm{md}}(k, \mu)=\left(1+\beta W(k) \mu^{2}\right)^{2} \exp \left\{-\left(k \mu \tilde{\sigma}_{v}\right)^{2}\right\} .
$$

We use two parameters to model the anisotropic measure, the RSD parameter $\beta$ and reduced velocity dispersion $\tilde{\sigma}_{v}$. Recall that we use $P_{g}^{s}(k, \mu=0)$ to estimate the realspace galaxy power spectrum and to measure the galaxy bias $b_{g} \sigma_{8}(z)$. This frees us from modeling nonlinearities in the galaxy power spectrum. In the appendix $\S \mathrm{A}$, we show with $N$-body simulation that neglecting high order corrections do not bias the $\beta$ constraint, for the adopted cut of $k<0.24 h / \mathrm{Mpc}$ and $k \mu \leq 0.1 h / \mathrm{Mpc}$.

\subsection{Covariance Matrix and Likelihood Analysis}

The covariance matrix is estimated using 1024 MDPatchy mocks,

$$
\begin{aligned}
C_{i j, k l}= & \frac{1}{N_{m}-1} \sum_{n=1}^{N_{m}}\left(\mathrm{AM}^{(n)}\left(k_{i}, \mu_{j}\right)-\widehat{\operatorname{AM}}\left(k_{i}, \mu_{j}\right)\right) \\
& \times\left(\mathrm{AM}^{(n)}\left(k_{k}, \mu_{l}\right)-\widehat{\operatorname{AM}}\left(k_{k}, \mu_{l}\right)\right)
\end{aligned}
$$

where $N_{m}=1024$ is the number of mock samples, $\operatorname{AM}^{(n)}\left(k_{i}, \mu_{j}\right)$ is the anisotropic measure for the $n$-th mock and $\widehat{\operatorname{AM}}\left(k_{i}, \mu_{j}\right)=\frac{1}{N_{m}} \sum_{n=1}^{N_{m}} \operatorname{AM}^{(n)}\left(k_{i}, \mu_{j}\right)$.

As shown by Hartlap et al. (2007), the inverse of the covariance matrix obtained above is a biased estimator of the true inverse covariance matrix. If the errors are Gaussian and data are statistically independent, we can rescale the obtained inverse covariance matrix by a constant factor to get unbiased inverse covariance matrix and log-likelihood function. The rescaling factor is $R_{1}=\left(N_{m}-N_{d}-2\right) /\left(N_{m}-1\right)$, where $N_{d}$ is the number of data points used in fitting procedure. 


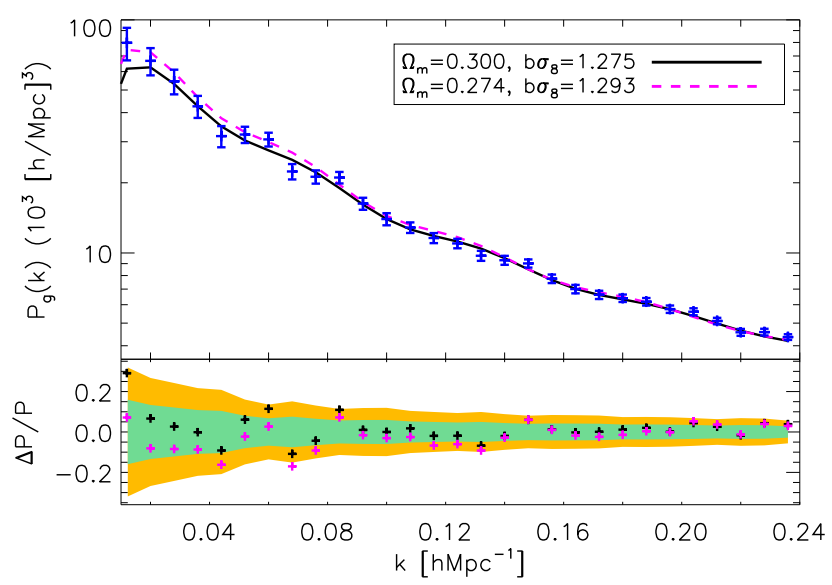

FIG. 5. - Real-space power spectrum of BOSS-DR11 CMASS galaxies. Upper panel: galaxy power spectrum in real space (black pluses with error bars estimated using 1024 MD-Patchy mocks, model power spectrum with bestfit $b \sigma_{8}\left(z_{\text {eff }}\right)$ for $\Omega_{m}=0.3$ (black solid line) and $\Omega_{m}=0.274$ (magenta dashed line). Lower panel: the fractional difference between model and observation for $\Omega_{m}=$ 0.3 (black pluses) and $\Omega_{m}=0.274$ (magenta pluses). The Celadon green (Chrome yellow) band show the $1 \sigma(2 \sigma)$ level of the fractional difference.

The error of the obtained covariance matrix also should be taken into account to estimate parameters from it. We rescale the variance of parameters by another constant factor as proposed by Percival et al. (2014), $R_{2}=\sqrt{\left(1+B\left(N_{d}-N_{p}\right) /\left(1+A+B\left(N_{p}+1\right)\right)\right.}$, where $A \approx 2\left(N_{m}-N_{d}-2.5\right)^{-2}$ and $B \approx\left(N_{m}-N_{d}-3\right)^{-1}$ if $N_{m}-N_{d} \gg 1$.

We assume the noise of the anisotropic measure is Gaussian distributed and we estimate the covariance matrix using mock samples. Then we construct the likelihood function as,

$$
\mathscr{L} \propto \exp \left\{-\chi^{2}(\mathbf{p}, \mathbf{d}) / 2\right\}
$$

where $\mathbf{p}$ is the parameter vector and $\mathbf{d}$ is data vector. The chi-square is defined as,

$$
\begin{aligned}
\chi^{2}(\mathbf{p}, \mathbf{d})= & \sum_{i j, k l}\left(\operatorname{AM}\left(k_{i}, \mu_{j}\right)-\operatorname{AM}^{\mathrm{md}}\left(k_{i}, \mu_{j}\right)\right) C_{i j, k l}^{-1} \\
& \times\left(\operatorname{AM}\left(k_{k}, \mu_{l}\right)-\mathrm{AM}^{\mathrm{md}}\left(k_{k}, \mu_{l}\right)\right)
\end{aligned}
$$

The model anisotropic measure $\mathrm{AM}^{\mathrm{md}}\left(k_{i}, \mu_{j}\right)$ is given in Eq. 12 with two parameters $\beta$ and $\tilde{\sigma}_{v} . C_{i j, k l}^{-1}$ is the inverse of covariance-matrix $C_{i j, k l}$ which is defined in Eq. 13 .

As discussed in Sec.5.3.1, we can measure the galaxy bias factor $b_{g} \sigma_{8}(z)$ from the real-space power spectrum. Combining the measurement of RSD parameter $\beta$ and galaxy bias factor, we can calculate the posterior distribution function of the structure growth rate by

$$
\mathcal{P}\left(f \sigma_{8}\right)=\int \mathcal{F}\left(b_{g} \sigma_{8}\right) \mathcal{G}\left(\beta=\frac{f \sigma_{8}}{b \sigma_{8}}\right) \frac{d\left(b_{g} \sigma_{8}\right)}{b_{g} \sigma_{8}} .
$$

Here $\mathcal{G}(\beta)$ is the posterior distribution function of RSD parameter $\beta$ and $\mathcal{F}\left(b_{g} \sigma_{8}\right)$ is the posterior distribution function of galaxy bias factor $b_{g} \sigma_{8}$. Notice that $f=f(z)$ and $\sigma_{8}=\sigma_{8}(z)$ in above equation.

The fiducial cosmology used to translate redshift to distance may differ from the true cosmology. This

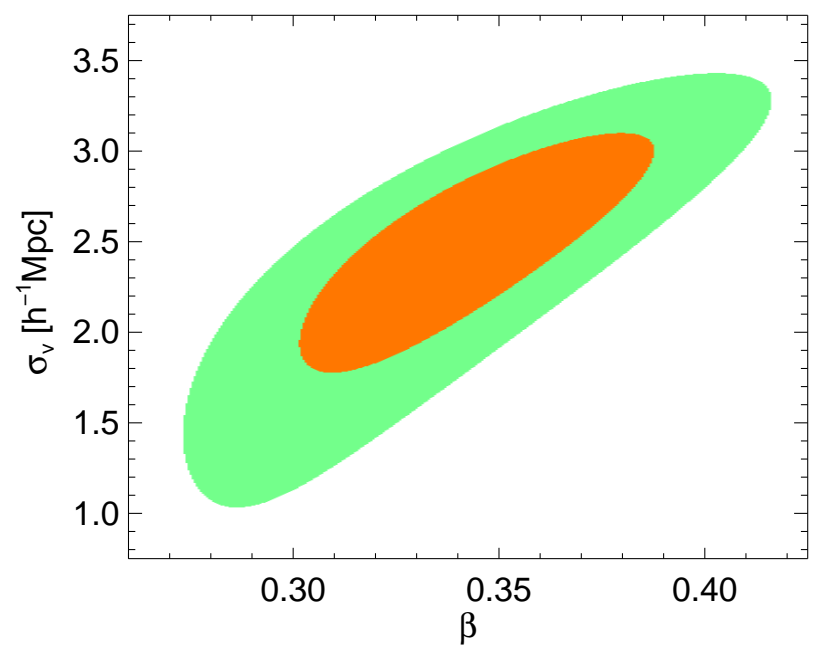

Fig. 6.- Two dimensional likelihood function of $\beta$ and $\tilde{\sigma}_{v}$ for BOSS-DR11 CMASS galaxies. The orange contours show $68 \%$ confidence level, while the green contours show $95 \%$ confidence level.

will introduce another distortion along the LOS direction and is called Alcock-Paczynski (AP) effect (Alcock \& Paczynski 1979; Li et al. 2014). As an illustration of the methodology, we neglect the AP effect in this work. We leave the detailed modeling of the RSD effect and the joint-analysis of anisotropic measure and real-space galaxy power spectrum to a future work.

\subsection{Measuring $f \sigma_{8}(z)$}

We obtain the constraints on the structure growth rate in three steps, namely the measurement of galaxy bias $b_{g} \sigma_{8}(z)$, RSD parameter $\beta$ and growth rate $f(z) \sigma_{8}(z)$.

\subsubsection{Galaxy Bias Factor}

We measure the combination of galaxy bias factor $b_{g}$ and $\sigma_{8}(z)$, which determines the amplitude of matter power spectrum, using the real-space galaxy power spectrum measurement $P_{g}(k)$. The real-space galaxy power spectrum can be obtained from the galaxy 2D power spectrum in the first $\mu$-bin $P_{g}(k)=P_{g}^{s}(k, \mu=0)$.

In this work, we fix the shape of matter power spectrum and adopt a single-parameter model for the galaxy power spectrum in real space,

$$
P_{g}(k)=\lambda^{2} \frac{P_{m, \mathrm{fid}}(k)}{\sigma_{8, \mathrm{fid}}^{2}\left(z_{\mathrm{eff}}\right)}
$$

where $\lambda=b_{g} \sigma_{8}\left(z_{\text {eff }}\right)$. We assume a $\Lambda$ CDM cosmology for the fiducial matter power spectrum $P_{m, \text { fid }}(k)$. Where the spectra index $n_{s}=0.96$ and Hubble parameter $h=0.7$. The baryon density parameter is given by $\Omega_{b}=\Omega_{m} / 6$ and $\Omega_{\Lambda}=1-\Omega_{m}$. The matter power spectrum is calculated using 'CAMB' (Lewis et al. 2000). The nonlinear evolution is corrected using 'halofit' model (Smith et al. 2003). To study the systematics introduced by the choice of $\Omega_{m}$ in the measurement of $b_{g} \sigma_{8}(z)$, we construct two different fiducial matter power spectrum, one with $\Omega_{m}=0.274$ (close to the WMAP7 bestfit value) and the other with $\Omega_{m}=0.3$ (close to the Planck2015 bestfit value).

We obtain $b_{g} \sigma_{8}\left(z_{\text {eff }}\right)=1.293 \pm 0.007$ for $\Omega_{m}=0.274$ and $b_{g} \sigma_{8}\left(z_{\text {eff }}\right)=1.274 \pm 0.007$ for $\Omega_{m}=0.3$. This shows 


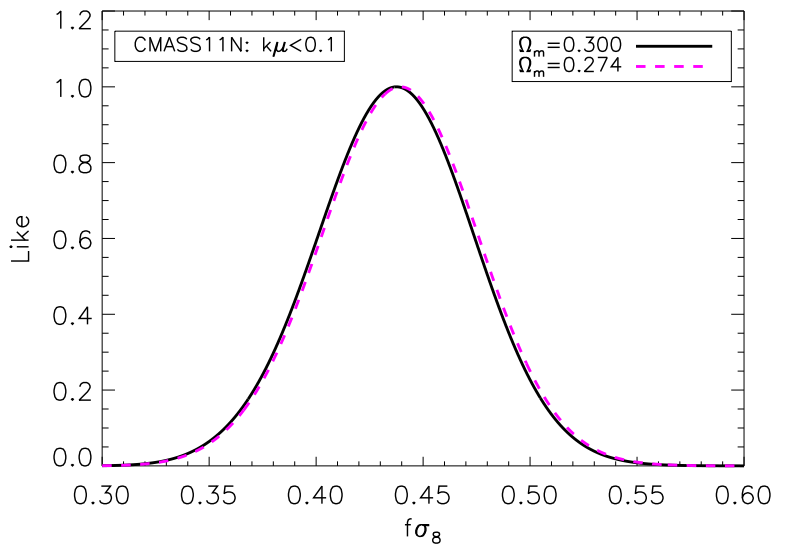

Fig. 7.- Normalized likelihood function of $f\left(z_{\mathrm{eff}}\right) \sigma_{8}\left(z_{\mathrm{eff}}\right)$ for BOSS-DR11 CMASS galaxies. The black solid curve shows results assuming $\Omega_{m}=0.3$ in measuring $b_{g} \sigma_{8}\left(z_{\text {eff }}\right)$ and the magenta dashed curve corresponds to $\Omega_{m}=0.274$. The figure shows negligible difference for these two cases.

that the measurement of $b_{q} \sigma_{8}\left(z_{\text {eff }}\right)$ is insensitive to the value of $\Omega_{m}$ in this model. The bestfit model power spectrum and the measured real-space power spectrum are shown in Fig. 5.

\subsubsection{Measurement of $\beta$}

Next, we do a maximum likelihood analysis to extract the RSD parameter $\beta$. The methods are discussed in Sec. 5.1. We use two parameters - RSD parameter $\beta$ and reduced velocity dispersion $\tilde{\sigma}_{v}$, to model the theoretical anisotropic measure. In this paper, we fix the $W(k)$ function (Eq.11) to reduce the number of parameters. The parameter $\Delta \alpha$ is calibrated in simulation (Zheng et al. 2013). We estimate the nonlinear matter power spectrum, which appears in $W(k)$, using the mean power spectrum of the 1024 MD-Patchy mocks.

We calculate the likelihood function on grids of $2 \mathrm{D}$ parameter space and take 1000 points for each parameter placed equally over range of $0.1<\beta<0.6$ for RSD parameter and $0<\tilde{\sigma}_{v}<10$ for velocity dispersion. The normalized two-dimensional likelihood function of $\beta$ and $\tilde{\sigma}_{v}$ are shown in Fig. 6 . The bestfit values are $\beta_{\text {best }}=0.3448$ and $\tilde{\sigma}_{v \text {,best }}=2.52 h^{-1} \mathrm{Mpc}$. We obtain the mean value and $1 \sigma$ error of $\beta=0.3403 \pm 0.0285$ and $\tilde{\sigma}_{v}=2.40 \pm 0.44 h^{-1} \mathrm{Mpc}$, by marginalizing over $\tilde{\sigma}_{v}$ and $\beta$ respectively.

\subsubsection{Measurement of $f\left(z_{\text {eff }}\right) \sigma_{8}\left(z_{\text {eff }}\right)$}

With the measurement of $\beta$ and $b_{g} \sigma_{8}\left(z_{\text {eff }}\right)$, we can obtain the growth rate of large scale structure $f\left(z_{\text {eff }}\right) \sigma_{8}\left(z_{\text {eff }}\right)$ through Eq. 16. We show the normalized likelihood function of $f\left(z_{\text {eff }}\right) \sigma_{8}\left(z_{\text {eff }}\right)$ in Fig. 7. Same as $b \sigma_{8}\left(z_{\text {eff }}\right)$, the result $f\left(z_{\text {eff }}\right) \sigma_{8}\left(z_{\text {eff }}\right)$ is insensitive to the value of $\Omega_{m}$. The bestfit value and $1 \sigma$ error of the structure growth rate is $f\left(z_{\text {eff }}\right) \sigma_{8}\left(z_{\text {eff }}\right)=0.440 \pm 0.037$ for $\Omega_{m}=0.274$ and $f\left(z_{\text {eff }}\right) \sigma_{8}\left(z_{\text {eff }}\right)=0.438 \pm 0.037$ for $\Omega_{m}=0.3$.

\section{DISCUSSIONS}

In Fig. 8, we present the reported measurements on $f\left(z_{\text {eff }}\right) \sigma_{8}\left(z_{\text {eff }}\right)$ at the same redshift of $z_{\text {eff }}=0.57$ (Chuang et al. 2013; Beutler et al. 2014; Samushia et al.

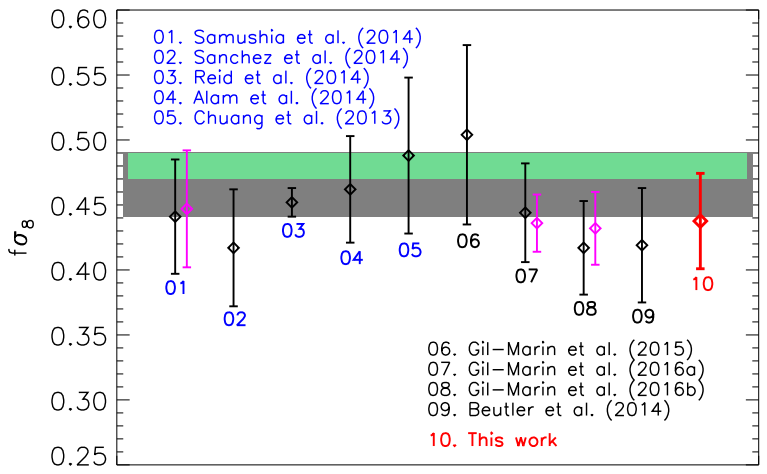

Fig. 8.- Constraints on $f\left(z_{\text {eff }}\right) \sigma_{8}\left(z_{\text {eff }}\right)$ from BOSS CMASS DR10, DR11 and DR12 release. Our result are shown in red diamond. Black diamonds show the results from various literatures. Magenta diamonds show those analysis that do not include the AP effect or use fiducial parameters for the AP effect. The green band show the $1 \sigma$ confidence level allowed by Planck 15 assuming $\Lambda \mathrm{CDM}+\mathrm{GR}$ model and grey band for WMAP9 assuming $\Lambda \mathrm{CDM}+\mathrm{GR}$ model.

2014; Sánchez et al. 2014; Reid et al. 2014; Alam et al. 2015; Gil-Marín et al. 2015a, 2016a,b). Here we give a brief summary on the data, statistics, model and the measured $f\left(z_{\text {eff }}\right) \sigma_{8}\left(z_{\text {eff }}\right)$ of these analysis. Notice that (1)-(5) are using correlation function measurements and (6)-(9) are using power spectrum measurements.

(1) Samushia et al. (2014) analyzed the monopole and quadrupole correlation function for BOSS-DR11 CMASS galaxies at scales of $24 h^{-1} \mathrm{Mpc}<s<$ $152 h^{-1} \mathrm{Mpc}$. The model correlation function are calculated using the 'streaming model'. They reported $f\left(z_{\text {eff }}\right) \sigma_{8}\left(z_{\text {eff }}\right)=0.441 \pm 0.044$ if the fitting method includes the AP effect and $f\left(z_{\text {eff }}\right) \sigma_{8}\left(z_{\text {eff }}\right)=$ $0.447 \pm 0.028$ if the AP effect is fixed.

(2) Sánchez et al. (2014) analyzed the correlation function monopole and wedges of BOSS-DR11 CMASS galaxies for $s \leq 40 h^{-1} \mathrm{Mpc}$. Through their model based on the renormalized perturbation theory, they obtained $f\left(z_{\text {eff }}\right) \sigma_{8}\left(z_{\text {eff }}\right)=0.417 \pm 0.045$.

(3) Reid et al. (2014) explored the anisotropic clustering of BOSS-DR10 CMASS galaxies on small scales of $0.8 \sim 32 h^{-1} \mathrm{Mpc}$. They reported a precise measurement of $f\left(z_{\text {eff }}\right) \sigma_{8}\left(z_{\text {eff }}\right)=0.450 \pm 0.011$.

(4) Alam et al. (2015) analyzed the monopole and quadrupole correlation function for the BOSS-DR11 CMASS galaxies at scales of $30 \sim 126 h^{-1} \mathrm{Mpc}$. Based on the Convolution Lagrangian Perturbation Theory with Gaussian streaming model, they obtained $f\left(z_{\text {eff }}\right) \sigma_{8}\left(z_{\text {eff }}\right)=0.462 \pm 0.041$.

(5) Chuang et al. (2013) reported a detection of $f\left(z_{\text {eff }}\right) \sigma_{8}\left(z_{\text {eff }}\right)=0.488 \pm 0.060$ by analyzing the monopole and quadrupole correlation function of BOSS-DR12 CMASS galaxies at scales of $55<s<$ $200 h^{-1} \mathrm{Mpc}$.

(6) Gil-Marín et al. (2015a) analyzed the monopole power spectrum and bispectrum of BOSS-DR11 
CMASS galaxies at scales up to $k_{\max }=0.17 \mathrm{hMpc}^{-1}$. They obtained $f\left(z_{\text {eff }}\right)^{0.43} \sigma_{8}\left(z_{\text {eff }}\right)=0.582 \pm 0.084$ without AP effect, which can be transformed to $f\left(z_{\text {eff }}\right) \sigma_{8}\left(z_{\text {eff }}\right)=0.504 \pm 0.069$ by using the fiducial value of $f_{\text {fid }}\left(z_{\text {eff }}\right)=0.777$.

(7) Gil-Marín et al. (2016a) analyzed the monopole and quadrupole power spectrum of BOSS-DR12 CMASS galaxies at scales up to $k_{\max }=0.24 \mathrm{hMpc}^{-1}$. The RSD effect are modeled based on Taruya et al. (2010). They obtained $f\left(z_{\text {eff }}\right) \sigma_{8}\left(z_{\text {eff }}\right)=0.444 \pm 0.038$ if AP effect is included in the fitting method and $f\left(z_{\text {eff }}\right) \sigma_{8}\left(z_{\text {eff }}\right)=0.436 \pm 0.022$ with fixed Hubble parameter and angular distance parameter.

(8) Gil-Marín et al. (2016b) improved the analysis of Gil-Marín et al. (2015a) by including more triangular shapes, using full covariance matrix, including quadrupole power spectrum and applying to BOSS-DR12 CMASS galaxies. Taking $k_{\max }=$ $0.22 h \mathrm{Mpc}^{-1}$, they obtained $f\left(z_{\text {eff }}\right) \sigma_{8}\left(z_{\text {eff }}\right)=0.417 \pm$ 0.027 with AP effect included in the fitting method and $f\left(z_{\text {eff }}\right) \sigma_{8}\left(z_{\text {eff }}\right)=0.432 \pm 0.028$ with fixed AP effect.

(9) Beutler et al. (2014) measured the monopole and quadrupole power spectrum for BOSS-DR11 CMASS galaxies at scales up to $k_{\max }=0.20 \mathrm{hMpc}^{-1}$. Their power spectrum model was based on Taruya et al. (2010) and the AP effect was included. They obtained $f\left(z_{\text {eff }}\right) \sigma_{8}\left(z_{\text {eff }}\right)=0.419 \pm 0.044$ and derived $\beta=0.342 \pm 0.037$.

It's worth noting that our measurements of $f\left(z_{\text {eff }}\right) \sigma_{8}\left(z_{\text {eff }}\right)$ are consistent with Samushia et al. (2014) at $1-2 \%$ level. Although our measurements of $\beta$ are consistent with Beutler et al. (2014) (within $1 \%)$, the values of $b \sigma_{8}\left(z_{\text {eff }}\right)$ are different by more than $4 \%$ which results in a more than $4 \%$ difference on $f\left(z_{\text {eff }}\right) \sigma_{8}\left(z_{\text {eff }}\right)$ measurement.

\section{CONCLUSION}

In this paper, we use the two-dimensional power spectrum in redshift space to measure the RSD effect. We revisit the method of measuring the galaxy $2 \mathrm{D}$ power spectrum by measuring and Fourier transforming the 2D correlation function. The $2 \mathrm{D}$ power spectrum measured in this way has several advantages: (A) they can improve the parallel-plane approximation and 'moving-LOS' approximation and capture all RSD information under the assumption of distant observer and neglecting wide-angle effect; (B) they are unbiased and free of normalization and shot-noise subtraction; $(\mathrm{C})$ the survey window function can be dealt with in configuration space; (D) the nonuniform distribution of cosine angle $\mu$ can be solved. Most importantly, working on 2D power spectrum opens the opportunity to separate the nonlinearities in the realto-redshift space mapping at data level.

We have tested the 2D power spectrum measurements using mock galaxies constructed from high resolution CosmicGrowth $N$-body simulation and concluded that our method can give unbiased measurement of $2 \mathrm{D}$ power spectrum for large galaxy surveys. After applying the method on the BOSS-DR11 CMASS galaxy sample, we report for the first time the measurement of $2 \mathrm{D}$ power spectrum for this sample.

We have introduced a new statistics, anisotropic measure, to extract the structure growth rate from the $2 \mathrm{D}$ power spectrum measurements. In this paper we used a simple model with two parameters $\beta$ and $\tilde{\sigma}_{v}$ to interpret this new measurement. We obtained $\beta=$ $0.3403 \pm 0.0285$ and $\tilde{\sigma}_{v}=2.40 \pm 0.44 h^{-1} \mathrm{Mpc}$ for BOSSDR11 CMASS galaxies. We further measured the galaxy bias factor from the real-space power spectrum, which is $b_{g} \sigma_{8}\left(z_{\text {eff }}\right)=1.274 \pm 0.007$. Combining the measurement of $\beta$ and $b_{g} \sigma_{8}\left(z_{\text {eff }}\right)$, we got the following measurement of the structure growth rate, $f\left(z_{\text {eff }}=0.57\right) \sigma_{8}\left(z_{\text {eff }}=\right.$ $0.57)=0.438 \pm 0.037$. This measurement together with the $2 \mathrm{D}$ power spectrum can be used to put interesting constraints on cosmological models. For this reason, we will release our results of $2 \mathrm{D}$ power spectrum soon.

\section{ACKNOWLEDGMENTS}

This work was supported by the National Science Foundation of China (Grants No. 11403071, 11320101002 \& 11533006) and National Basic Research Program of China (973 Programs No. 2015CB857001 \& 2015CB857003). ZL is supported by China Postdoctoral Science Foundation Funded Project (No. 2013M541511).

We acknowledge the BOSS collaboration to kindly release the BOSS DR11 CMASS galaxy sample and the MD-Patchy mock sample plubicly. Funding for SDSS-III has been provided by the Alfred P. Sloan Foundation, the Participating Institutions, the National Science Foundation, and the U.S. Department of Energy Office of Science. The SDSS-III web site is http://www.sdss3.org/.

\section{REFERENCES}

Alam, S., Ho, S., Vargas-Magaña, M., \& Schneider, D. P. 2015, MNRAS, 453, 1754, 1504.02100

Alcock, C., \& Paczynski, B. 1979, Nature, 281, 358

Amendola, L., Quercellini, C., \& Giallongo, E. 2005, MNRAS, 357,429 , astro-ph/0404599

Anderson, L. et al. 2014, MNRAS, 441, 24, 1312.4877

Beutler, F. et al. 2012, MNRAS, 423, 3430, 1204.4725 2014, MNRAS, 443, 1065, 1312.4611

Blake, C. et al. 2011, MNRAS, 415, 2876, 1104.2948 2016, MNRAS, 456, 2806, 1507.03086

Cabré, A., \& Gaztañaga, E. 2009a, MNRAS, 393, 1183, 0807.2460 2009b, MNRAS, 396, 1119, 0807.2461

Cai, Y.-C., \& Bernstein, G. 2012, MNRAS, 422, 1045, 1112.4478
Chuang, C.-H., Kitaura, F.-S., Liang, Y., Font-Ribera, A., Zhao, C., McDonald, P., \& Tao, C. 2016, ArXiv e-prints, 1605.05352 Chuang, C.-H. et al. 2013, ArXiv e-prints, 1312.4889

Cole, S., Fisher, K. B., \& Weinberg, D. H. 1995, MNRAS, 275, 515, astro-ph/9412062

Cuesta, A. J. et al. 2016, MNRAS, 457, 1770, 1509.06371

Dodelson, S., Heitmann, K., Hirata, C., Honscheid, K., Roodman, A., Seljak, U., Slosar, A., \& Trodden, M. 2016, ArXiv e-prints, 1604.07626

Erdoğdu, P. et al. 2006, MNRAS, 373, 45, astro-ph/0610005

Feldman, H. A., Kaiser, N., \& Peacock, J. A. 1994, ApJ, 426, 23, astro-ph/9304022

Feng, J. L. et al. 2014, ArXiv e-prints, 1401.6085 
Fisher, K. B., Davis, M., Strauss, M. A., Yahil, A., \& Huchra, J. P. 1994, MNRAS, 267, 927, astro-ph/9308013

Fisher, K. B., Lahav, O., Hoffman, Y., Lynden-Bell, D., \& Zaroubi, S. 1995, MNRAS, 272, 885, astro-ph/9406009

Gil-Marín, H., Noreña, J., Verde, L., Percival, W. J., Wagner, C., Manera, M., \& Schneider, D. P. 2015a, MNRAS, 451, 539, 1407.5668

Gil-Marín, H. et al. 2016a, MNRAS, 1509.06386

Gil-Marín, H., Percival, W. J., Verde, L., Brownstein, J. R., Chuang, C.-H., Kitaura, F.-S., Rodríguez-Torres, S. A., \& Olmstead, M. D. 2016b, ArXiv e-prints, 1606.00439

Gil-Marín, H. et al. 2015b, MNRAS, 452, 1914, 1408.0027

Guzzo, L. et al. 2008, Nature, 451, 541, 0802.1944

Hamilton, A. J. S. 1993, ApJ, 417, 19

Hamilton, A. J. S., \& Tegmark, M. 2004, MNRAS, 349, 115, astro-ph/0306324

Hamilton, A. J. S., Tegmark, M., \& Padmanabhan, N. 2000 MNRAS, 317, L23, astro-ph/0004334

Han, J., Jing, Y. P., Wang, H., \& Wang, W. 2012, MNRAS, 427, 2437, 1103.2099

Hartlap, J., Simon, P., \& Schneider, P. 2007, A\&A, 464, 399, astro-ph/0608064

Heavens, A. F., \& Taylor, A. N. 1995, MNRAS, 275, 483, astro-ph/9409027

Hikage, C., \& Yamamoto, K. 2013, JCAP, 8, 019, 1303.3380

Howlett, C., Ross, A. J., Samushia, L., Percival, W. J., \& Manera, M. 2015, MNRAS, 449, 848, 1409.3238

Jain, B., \& Zhang, P. 2008, Phys. Rev. D, 78, 063503, 0709.2375

Jennings, E., Baugh, C. M., \& Pascoli, S. 2011, ApJ, 727, L9, 1011.2842

Jing, Y. P., \& Börner, G. 2001, MNRAS, 325, 1389, astro-ph/0101211

- 2004, ApJ, 617, 782, astro-ph/0406077

Jing, Y. P., Suto, Y., \& Mo, H. J. 2007, ApJ, 657, 664, astro-ph/0610099

Johnson, A., Blake, C., Dossett, J., Koda, J., Parkinson, D., \& Joudaki, S. 2016, MNRAS, 458, 2725, 1504.06885

Kaiser, N. 1987, MNRAS, 227, 1

Kanemaru, T., Hikage, C., Hütsi, G., Terukina, A., \& Yamamoto, K. 2015, Phys. Rev. D, 92, 023523, 1501.04372

Kitaura, F.-S., Gil-Marín, H., Scóccola, C. G., Chuang, C.-H., Müller, V., Yepes, G., \& Prada, F. 2015, MNRAS, 450, 1836, 1407.1236

Kitaura, F.-S., \& Heß, S. 2013, MNRAS, 435, L78, 1212.3514

Kitaura, F.-S. et al. 2016, MNRAS, 456, 4156, 1509.06400

Kitaura, F.-S., Yepes, G., \& Prada, F. 2014, MNRAS, 439, L21, 1307.3285

Landy, S. D., Shectman, S. A., Lin, H., Kirshner, R. P., Oemler, A. A., \& Tucker, D. 1996, ApJ, 456, L1, astro-ph/9510146

Lanusse, F., Rassat, A., \& Starck, J.-L. 2012, A\&A, 540, A92, 1112.0561

- 2015, A\&A, 578, A10, 1406.5989

Leistedt, B., Rassat, A., Réfrégier, A., \& Starck, J.-L. 2012, A\&A, 540, A60, 1111.3591

Leonard, C. D., Ferreira, P. G., \& Heymans, C. 2015, JCAP, 12, $051,1510.04287$

Lewis, A., Challinor, A., \& Lasenby, A. 2000, ApJ, 538, 473, astro-ph/9911177

Li, X.-D., Park, C., Forero-Romero, J. E., \& Kim, J. 2014, ApJ, $796,137,1412.3564$

Linder, E. V. 2008, Astroparticle Physics, 29, 336, 0709.1113

Loveday, J., Efstathiou, G., Maddox, S. J., \& Peterson, B. A. 1996, ApJ, 468, 1, astro-ph/9505099

Oka, A., Saito, S., Nishimichi, T., Taruya, A., \& Yamamoto, K. 2014, MNRAS, 439, 2515, 1310.2820

Okumura, T. et al. 2016, PASJ, 68, 38, 1511.08083
Okumura, T., Matsubara, T., Eisenstein, D. J., Kayo, I., Hikage, C., Szalay, A. S., \& Schneider, D. P. 2008, ApJ, 676, 889, 0711.3640

Park, C., Vogeley, M. S., Geller, M. J., \& Huchra, J. P. 1994, ApJ, 431, 569

Peacock, J. A. et al. 2001, Nature, 410, 169, astro-ph/0103143

Percival, W. J. et al. 2004, MNRAS, 353, 1201, astro-ph/0406513

- 2014, MNRAS, 439, 2531, 1312.4841

Percival, W. J., \& White, M. 2009, MNRAS, 393, 297, 0808.0003

Pullen, A. R., Alam, S., He, S., \& Ho, S. 2015a, ArXiv e-prints, 1511.04457

Pullen, A. R., Alam, S., \& Ho, S. 2015b, MNRAS, 449, 4326, 1412.4454

Rassat, A., \& Refregier, A. 2012, A\&A, 540, A115, 1112.3100

Ratcliffe, A., Shanks, T., Parker, Q. A., \& Fong, R. 1998,

MNRAS, 296, 191, astro-ph/9702228

Reid, B. A. et al. 2012, MNRAS, 426, 2719, 1203.6641

Reid, B. A., Seo, H.-J., Leauthaud, A., Tinker, J. L., \& White, M. 2014, MNRAS, 444, 476, 1404.3742

Reyes, R., Mandelbaum, R., Seljak, U., Baldauf, T., Gunn, J. E.,

Lombriser, L., \& Smith, R. E. 2010, Nature, 464, 256, 1003.2185

Ross, A. J. et al. 2012, MNRAS, 424, 564, 1203.6499

. 2014, MNRAS, 437, 1109, 1310.1106

Samushia, L., Percival, W. J., \& Raccanelli, A. 2012, MNRAS, 420, 2102, 1102.1014

Samushia, L. et al. 2014, MNRAS, 439, 3504, 1312.4899

Sánchez, A. G. et al. 2014, MNRAS, 440, 2692, 1312.4854

Seljak, U., \& Zaldarriaga, M. 1996, ApJ, 469, 437, astro-ph/9603033

Smith, R. E. et al. 2003, MNRAS, 341, 1311, astro-ph/0207664

Song, Y.-S., \& Percival, W. J. 2009, JCAP, 10, 004, 0807.0810

Swanson, M. E. C., Tegmark, M., Hamilton, A. J. S., \& Hill, J. C. 2008, MNRAS, 387, 1391, 0711.4352

Tadros, H. et al. 1999, MNRAS, 305, 527, astro-ph/9901351

Taruya, A., Nishimichi, T., \& Saito, S. 2010, Phys. Rev. D, 82, 063522, 1006.0699

Taylor, A. N., Ballinger, W. E., Heavens, A. F., \& Tadros, H. 2001, MNRAS, 327, 689

Tegmark, M. et al. 2004, ApJ, 606, 702, astro-ph/0310725

Tegmark, M., Hamilton, A. J. S., \& Xu, Y. 2002, MNRAS, 335, 887, astro-ph/0111575

Wang, L., \& Jing, Y. P. 2010, MNRAS, 402, 1796, 0911.1864

Wang, L., Li, C., Kauffmann, G., \& De Lucia, G. 2006, MNRAS, 371, 537, astro-ph/0603546

Wang, Y. 2008, JCAP, 5, 021, 0710.3885

White, M., Song, Y.-S., \& Percival, W. J. 2009, MNRAS, 397, $1348,0810.1518$

Yamamoto, K., Bassett, B. A., \& Nishioka, H. 2005, Physical Review Letters, 94, 051301, astro-ph/0409207

Yamamoto, K., Nakamichi, M., Kamino, A., Bassett, B. A., \& Nishioka, H. 2006, PASJ, 58, 93, astro-ph/0505115

Yamamoto, K., Nakamura, G., Hütsi, G., Narikawa, T., \& Sato, T. 2010, Phys. Rev. D, 81, 103517, 1004.3231

Yamamoto, K., Sato, T., \& Hütsi, G. 2008, Progress of

Theoretical Physics, 120, 609, 0805.4789

Yoo, J., \& Seljak, U. 2015, MNRAS, 447, 1789, 1308.1093

Zhang, P., Liguori, M., Bean, R., \& Dodelson, S. 2007, Physical Review Letters, 99, 141302, 0704.1932

Zhang, P., Pan, J., \& Zheng, Y. 2013, Phys. Rev. D, 87, 063526, 1207.2722

Zheng, Y., \& Song, Y.-S. 2016, ArXiv e-prints, 1603.00101

Zheng, Y., Zhang, P., Jing, Y., Lin, W., \& Pan, J. 2013,

Phys. Rev. D, 88, 103510, 1308.0886

\section{APPENDIX}

\section{A. TESTing SyStematiCS USING N-BODY Simulation: VALUE OF $K_{\text {MAX }}$}

The nonlinearities related to the real-to-redshift space mapping are important at large $\mu$ and large $k$ (Zheng \& Song 2016). In Sec. 5.1, we mentioned that to suppress the effect of nonlinearities in the real-to-redshift space mapping, we would like to use data with the wavenumber $k$ and $k \mu$ smaller than some maximum value. Following the work of Zheng \& Song (2016), we adopt $k \mu_{\max }=0.1 \mathrm{hMpc}^{-1}$ for the BOSS-DR11 CMASS galaxies. Now we determine the value of $k_{\max }$ using the mock galaxy catalog in the CosmicGrowth $N$-body simulation. 


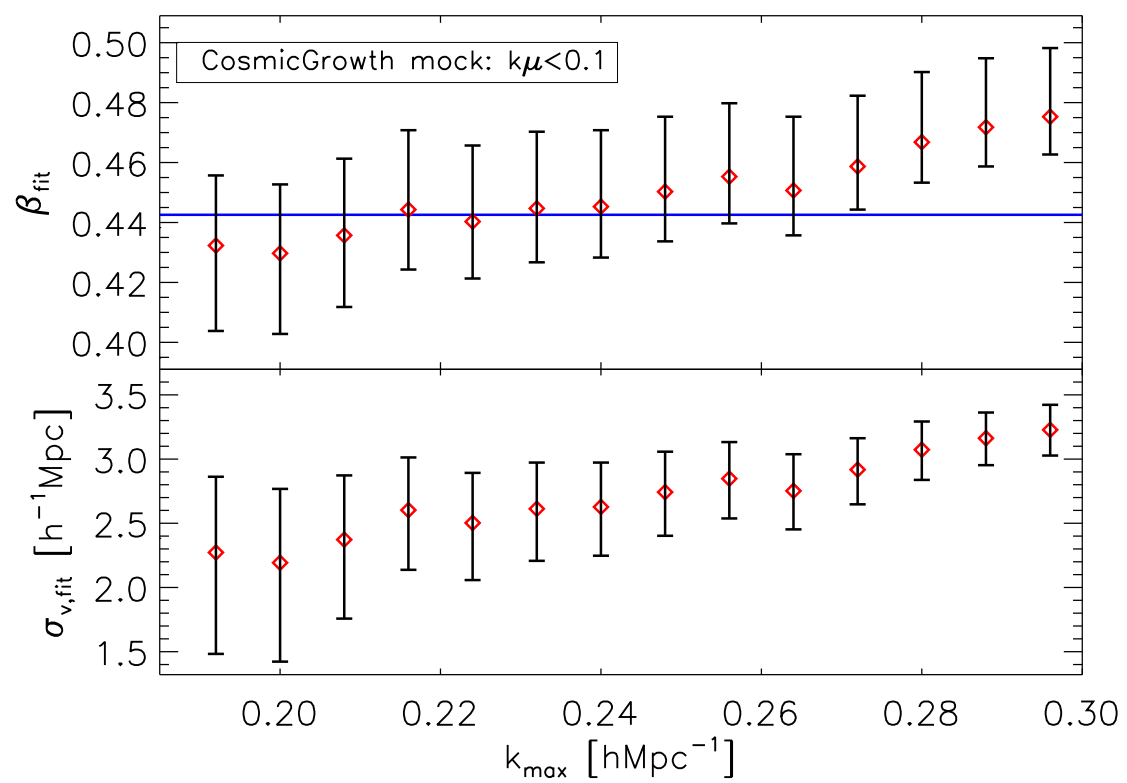

Fig. 9.- Bestfit value of $\beta$ and $\tilde{\sigma}_{v}$ as a function of $k_{\max }$ for mock galaxies in CosmicGrowth simulation. Upper panel: red diamonds are bestfit values of $\beta$, black bars are the $68 \%$ confidence region of $\beta$ marginalizing over $\tilde{\sigma}_{v}$. Blue solid line shows the prediction of General Relativity with $\Omega_{m}=0.268$ and $b_{\text {gal }}=1.68$. Lower panel: same as upper panel, but for $\tilde{\sigma}_{v}$.

We obtained the anisotropic measure for the mock galaxy catalog following the procedure described in the main text. We assume a diagonal covariance matrix including the cosmic variance and shot noise. Next we do the likelihood analysis by using different values of $k_{\max }$. The bestfit value of $\beta$ and $\tilde{\sigma}_{v}$ as a function of $k_{\max }$ is shown in Fig. 9 . The $68 \%$ confidence region of $\beta\left(\tilde{\sigma}_{v}\right)$ is obtained by marginalizing over $\tilde{\sigma}_{v}(\beta)$. Fig. 9 suggests that $k_{\max }$ should be no more than $0.24 h \mathrm{Mpc}^{-1}$ to get unbiased measurement of $\beta$. Taking $k_{\max }$ greater than $0.24 h \mathrm{Mpc}^{-1}$ we will obtain positively biased estimate of $\beta$ which shows the nonlinear effect in real-to-redshift space mapping. Concerning the large statistical error, one would use $k_{\max }=0.27 \mathrm{hMpc}^{-1}$ and the induced bias on $\beta$ is still within $1 \sigma$ region of GR prediction. To be conservative, we suggest to use $k_{\max }=0.24 \mathrm{hMpc}^{-1}$. In the lower panel of Fig. 9 , we show the bestfit value of $\tilde{\sigma}_{v}$ as a function of $k_{\max }$, which is a constant for our adopted $k_{\max }$

\section{B. FURTHER CONSISTENT CHECKS AGAINST EXISTING BOSS MEASUREMENTS}

Our correlation function based method of measuring the 2D power spectrum has been verified against simulations. Here we show further consistency tests.

\section{B.1. Further consistent check: Recovered Correlation Function}

If our measured power spectrum is correct, with it one must be able to recover the measured correlation function by the BOSS collaborations. Therefore we calculate the monopole and quadrupole correlation from the power spectrum that we measured, by

$$
\xi_{l}(s)=i^{l} \int P_{l}(k) j_{l}(k s) k^{2} \frac{d k}{2 \pi^{2}} .
$$

Here $j_{l}(x)$ is the spherical Bessel function, $j_{0}(x)=\sin (x) / x$ and $j_{2}(x)=\left(3 / x^{2}-1\right) \sin (x) / x-3 \cos (x) / x^{2}$. We show them in Fig. 10, against the measurements reported in literatures of Anderson et al. (2014), Samushia et al. (2014) and Sánchez et al. (2014). The figure shows that we can successfully recover the monopole and quadrupole correlation function without any notable bias.

\section{B.2. Multipole Power Spectrum: CMASS Galaxies}

The BOSS collaboration has already measured the 2D power spectrum monopole and quadrupole (Beutler et al. 2014; Anderson et al. 2014). These measurements have not been corrected the window function effect. Therefore to compare with these results, we need to convolve the monopole and quadrupole that we measured, with the appropriate window function,

$$
P_{l}^{\text {conv }}(k)=2 \pi \sum_{l^{\prime}} \int d k^{\prime} k^{\prime 2} P_{l^{\prime}}\left(k^{\prime}\right)\left|W\left(k, k^{\prime}\right)\right|_{l l^{\prime}}^{2} .
$$

Here, $\left|W\left(k, k^{\prime}\right)\right|_{l l^{\prime}}^{2}$ is the BOSS-DR11 CMASS window function, provided in Beutler et al. (2014). In light of the large effective volume of BOSS-DR11 CMASS sample, the window function only have small effect at very large scales and 


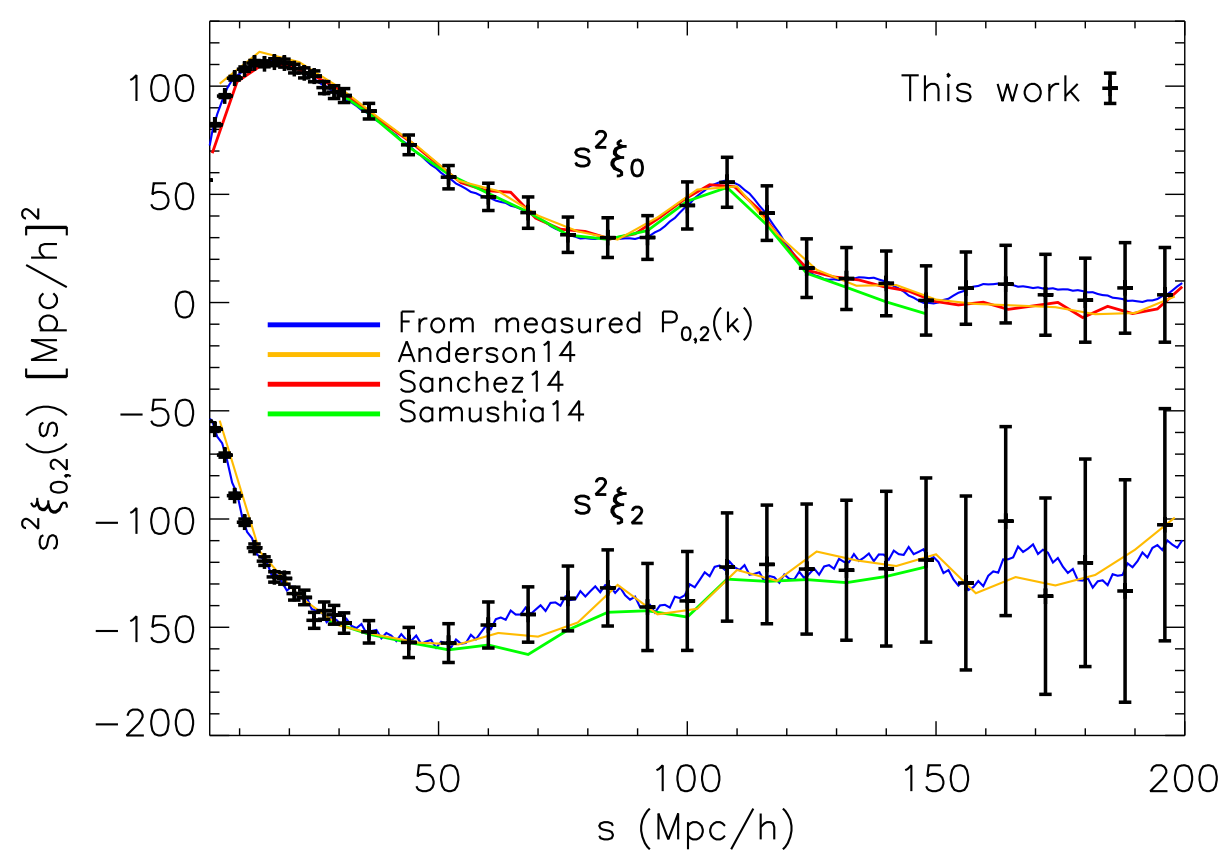

FIG. 10.- Monopole and quadrupole correlation function of BOSS-DR11 CMASS galaxies. Top of the figure show the monopole correlation function $s^{2} \xi_{0}(s)$, while bottom show quadrupole correlation function $s^{s} \xi_{2}(s)$ which have been shifted downward by a value of 80. Black plus symbols represent our measurements using Landy-Szalay estimator. Errors are estimated using MD-Patchy mocks. Blue solid lines show the correlation function calculated from the measured power spectrum $P_{0,2}(k)$ using Eq. B1, respectively. We also show the measurements in literatures: brown solid lines for Anderson et al. (2014), red solid lines for Sánchez et al. (2014) and blue solid lines for Samushia et al. (2014).

can be neglected at smaller scales. Here, we do not correct for the integral constraint effect, since both methods suffer from it.

The comparisons are shown in Fig. 11 for monopole power spectrum and in Fig. 12 for quadrupole power spectrum. We find that the convolved monopole power power spectrum match the measurements in Beutler et al. (2014) and in Anderson et al. (2014) very well, up to a scaling factor $\eta=0.90\left(P_{l}^{\text {conv }}(k) \rightarrow \eta P_{l}^{\text {conv }}(k)\right)$. Namely our power spectrum is about $10 \%$ higher than theirs. Interestingly, by the same scaling, the convolved quadrupole power spectrum also match the measurements in Beutler et al. (2014). Such bias factor can result in about $4 \%$ difference on the estimated $b_{g} \sigma_{8}$ and then on the growth rate $f \sigma_{8}$, although we obtained the same anisotropy measurement on the galaxy clustering as in Beutler et al. (2014) or the same $\beta(\S 5)$. We do not know the origin of such bias at this moment. We also notice that the monopole power spectra in Beutler et al. (2014) and Anderson et al. (2014) show discrepancies too and differ by a shot-noise-like term of constant amplitude. Nevertheless, our measurements of monopole/quadrupole power spectrum and monopole/quadrupole correlation function are self-consistent, as shown in previous subsection $\S \mathrm{B} .1$. 


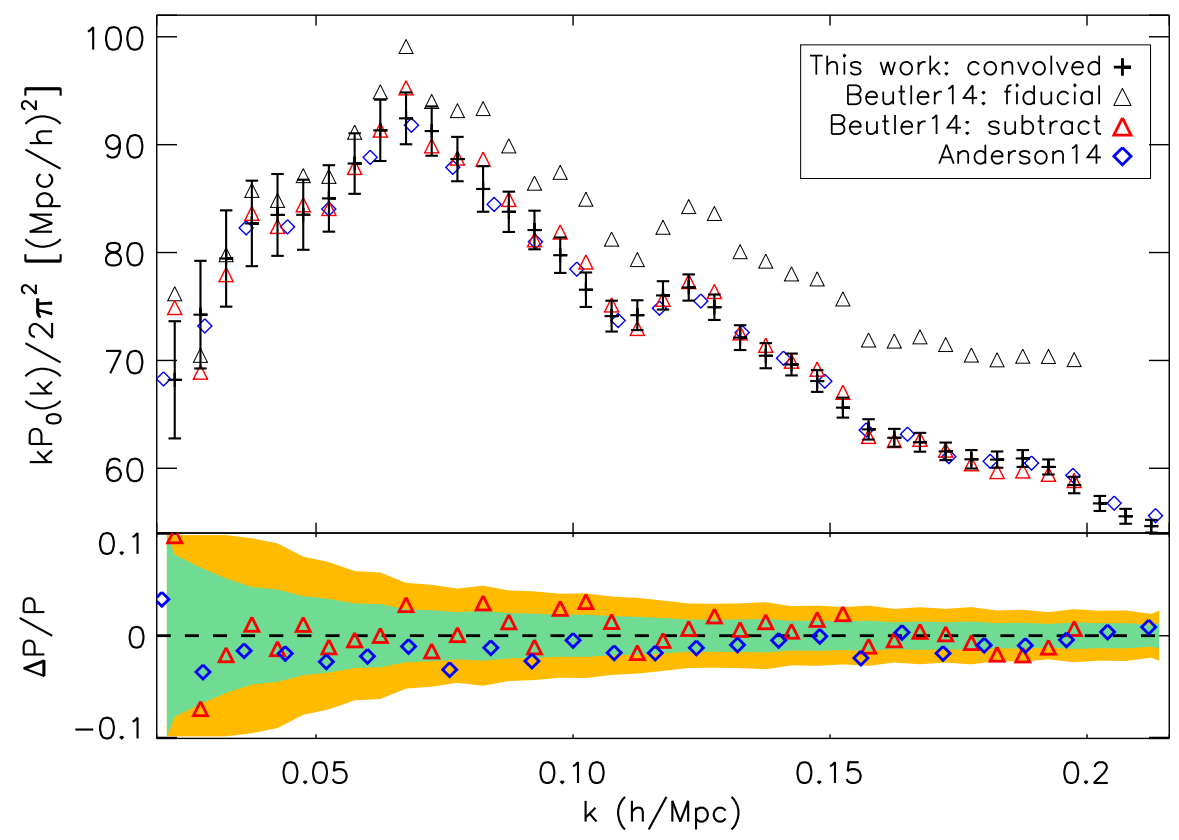

FIG. 11.- Monopole power spectrum of BOSS-DR11 CMASS NGC galaxies. Top panel: monopole power spectrum, $k P_{0}(k) / 2 \pi^{2}$, in our analysis after being convolved with window function and rescaled by $\eta=0.90$ (black plus symbols), in Beutler et al. (2014) (black triangles), in Anderson et al. (2014) (blue diamonds) and in Beutler et al. (2014) after being subtracted a constant value of 1120 on $P_{0}^{\mathrm{B} 14}(k)$ (red triangles). Bottom panel: difference between our analysis and those in literatures, $\Delta P / P=\left(\tilde{P}_{0}(k)-P_{0}(k)\right) / P_{0}(k)$ where $\tilde{P}_{0}(k)=P_{0}^{\mathrm{B} 14}(k)-1120$ for Beutler et al. (2014) and $\tilde{P}_{0}(k)=P_{0}^{\mathrm{A} 14}(k)$ for Anderson et al. (2014). Errors in our analysis are calculated using 1024 MD-Patchy mocks, also being convolved and rescaled in a way same as in observation.

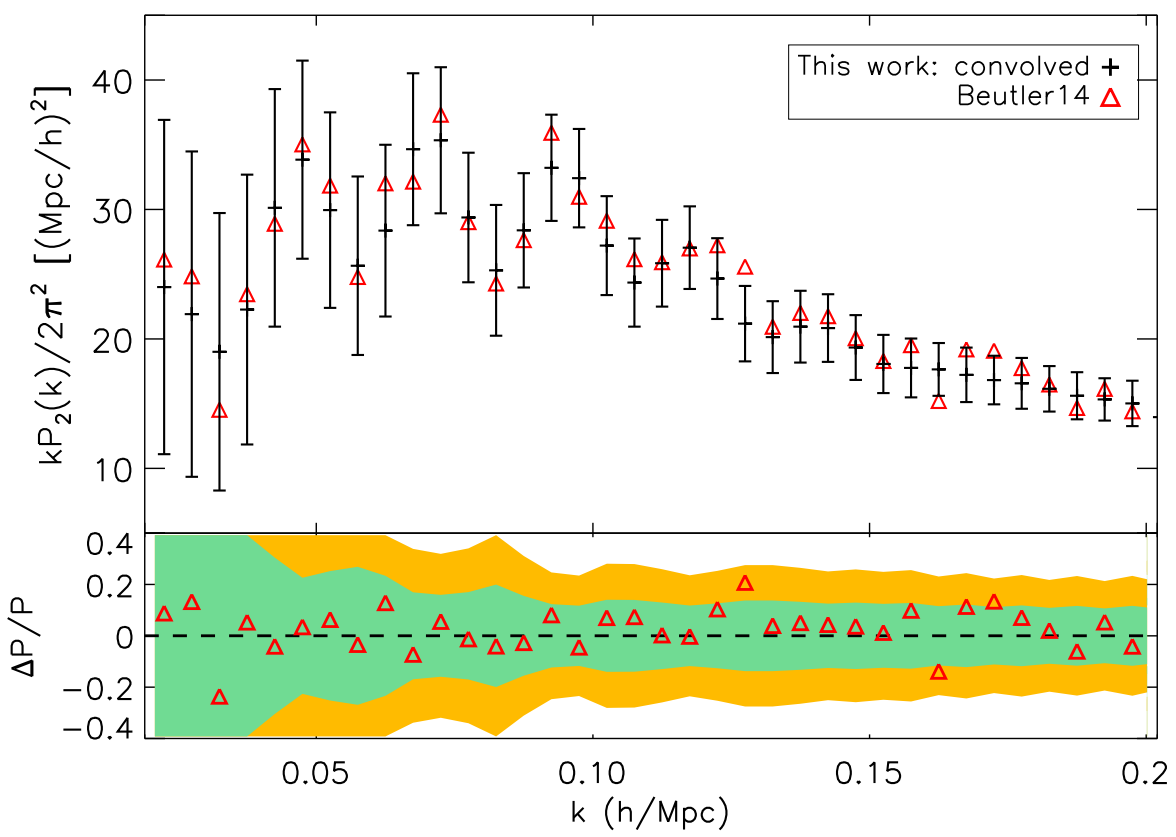

FIG. 12.- Quadrupole power spectrum of BOSS-DR11 CMASS NGC galaxies. Top panel: quadrupole power spectrum, $k P_{2}(k) / 2 \pi^{2}$, in our analysis (black plus symbols) and in Beutler et al. (2014) (red triangles). Bottom panel: difference between our analysis and those in Beutler et al. (2014), $\Delta P / P=\left(P_{2}^{\mathrm{B} 14}(k)-P_{2}(k)\right) / P_{2}(k)$. 